\title{
8. CR-RICH SPINEL AS A TRACER FOR MELT MIGRATION AND MELT-WALL ROCK INTERACTION IN THE MANTLE: HESS DEEP, LEG $147^{1}$
}

\author{
James F. Allan ${ }^{2}$ and Henry J.B. Dick ${ }^{3}$
}

\begin{abstract}
Site 895 of the Ocean Drilling Program Leg 147 cored melt-impregnated peridotite from the southeast flank of the Hess Deep intrarift high, recovering generalized stratigraphic sequences of harzburgite-dunite-troctolite-gabbro-troctolite-duniteharzburgite, with the gabbros representing fossil melt conduits through preexisting harzburgite. $\mathrm{Cr}$-rich spinel within the Site 895 samples reflects and records the effects of these multiple injections of melt into depleted, residual harzburgites of the East Pacific Rise uppermost mantle. Gabbroic spinel compositions vary widely $\left(\mathrm{TiO}_{2}, 0.55 \%-2.56 \% ; \mathrm{Mg} /\left[\mathrm{Mg}+\mathrm{Fe}^{2+}\right], 0.30-0.60\right)$, showing that the impregnating melts became locally quite evolved, with variation in melt composition controlled by both crystal fractionation and local melt-rock interaction. $\mathrm{TiO}_{2}$ contents in associated dunitic spinel are 10-20 times as high as in harzburgitic spinels $(0.5 \%-1.1 \%$ vs. $\leq 0.07 \%$, respectively), suggesting that the dunite was produced by melt-wall rock interaction associated with melt impregnation. This process produced halos of dunite around residual bodies of gabbroic rock and possible residual dunite "trails" of melt migration through peridotite. Spinel and other phase compositional data are consistent with the injection of depleted, exotic N-MORB melt by both diking and intergranular flow at depths shallower than $10 \mathrm{~km}$ and temperatures well above $900^{\circ} \mathrm{C}$, and most likely approaching about $1230^{\circ} \mathrm{C}$. High $\mathrm{ZnO}$ (to $0.75 \%$ ) in the metamorphically altered rims of spinel in gabbro and troctolite indicates that $\mathrm{Zn}$-rich hydrothermal fluids may have been present during post-crystallization, lower amphibolite to zeolite facies metamorphic alteration of the Hess Deep peridotites, with the gabbroic melt impregnations providing possible preferential conduits for fluid flow.
\end{abstract}

\section{INTRODUCTION}

Processes of melt and fluid generation, transportation, storage, and crystallization in the oceanic upper mantle and crust are at present imperfectly understood, with progress dependent on recovery of samples that preserve stratigraphic relations. Leg 147 of the Ocean Drilling Program (ODP) represented the first leg planned around the strategy of "offset drilling," designed to create a composite section of oceanic crust and uppermost mantle by drilling a number of holes in crustal sections exposed by faulting. During Leg 147, Sites 894 and 895 were cored on the summit and southeast flank of the Hess Deep intrarift high, a north-tilted fault block within Hess Deep, a 2500-mdeep fault basin at the tip of the westward-propagating Cocos-Nazca spreading center (Fig. 1; Lonsdale, 1988; Hekinian et al., 1993; Francheteau et al., 1990; Gillis, Mével, Allan, et al., 1993). Drilling at these sites recovered sections of mid-crustal gabbroic rocks and uppermost mantle peridotite, respectively (Gillis, Mével, Allan, et al., 1993).

The recovery from the six Site 895 holes consists of peridotite that has been impregnated by basaltic melt at a depth most likely near the crust/mantle boundary (Gillis, Mével, Allan, et al., 1993; Fig. 2). Although rocks of similar nature have been recovered from the Hess Deep (Hekinian et al., 1993; Girardeau and Francheteau, 1993), the Site 895 samples are of greater petrological value owing to their

'Mével, C., Gillis, K.M., Allan, J.F., and Meyer, P.S. (Eds.), 1996. Proc. ODP. Sci, Results, 147: College Station, TX (Ocean Drilling Program).

${ }^{2}$ Ocean Drilling Program, Texas A\&M University Research Park, 1000 Discovery Drive, College Station, TX 77845-9547, U.S.A., and Department of Geology and Geophysics, Texas A\&M University, College Station, TX, 77843, U.S.A. James_Allan@odp.tamu.edu

${ }^{3}$ Department of Geology and Geophysics, Woods Hole Oceanographic Institution, Woods Hole, MA 02543, U.S.A. hdick@ whoi.edu fresher nature and to the preservation of stratigraphic relations within the core. Although complicated by imperfect recovery and hole location in what might be rotated fault slumps (Kelso et al., this volume; Kikawa et al., this volume), the recovered core nevertheless shows fairly consistent local stratigraphic assemblages of harzburgite-dunite-troctolite-gabbroic rock-troctolite-dunite-harzburgite. The intimate association of large amounts of gabbroic rock with dunite in core is similar to what has been observed in ophiolites (Nicolas, 1989) and is exemplified by the comparative recoveries of Holes $895 \mathrm{D}$ and $895 \mathrm{E}$ (Fig. 3). The gabbroic and troctolitic rocks represent melt injection, by diking and intergranular flow (perhaps on dike margins), into host peridotite at shallow mantle levels (Gillis, Mével, Allan, et al., 1993; Hekinian et al., 1993; Girardeau and Francheteau, 1993; Dick, 1989; Nicolas, 1989). The sporadic presence of contact relations within the gabbroic rocks (Fig. 4, showing an olivine gabbro crosscutting a troctolite) underscores the interpretation of the Leg 147 shipboard party that melt injection into these peridotites occurred in multiple events (Gillis, Mével, Allan, et al., 1993).

$\mathrm{Cr}$-rich spinel is a ubiquitous phase in nearly all Site 895 rock. It is a highly sensitive indicator of a wide variety of petrogenetic processes (Dick and Bullen, 1984; Allan et al., 1988), recording variations in host melt and coexisting phase chemistry, temperature, and $\mathrm{fO}_{2}$. In this paper, we use high-precision analyses of spinel within these samples to track processes of mass transfer during basalt melt impregnation, and use this data to address two of the most vexing problems of mid-ocean-ridge basalt (MORB) petrogenesis: the nature of melt transport and the degree of melt-wall rock interaction in the upper mantle beneath spreading ridges. We focus on reporting spinel core-to-rim transects of major and minor element variation, examining the effects of basaltic melt-wall rock interaction on both the impregnating melt and the surrounding peridotite wall rock, exploring the origin of mantle dunite, and placing constraints on the physical conditions of melt impregnation. 


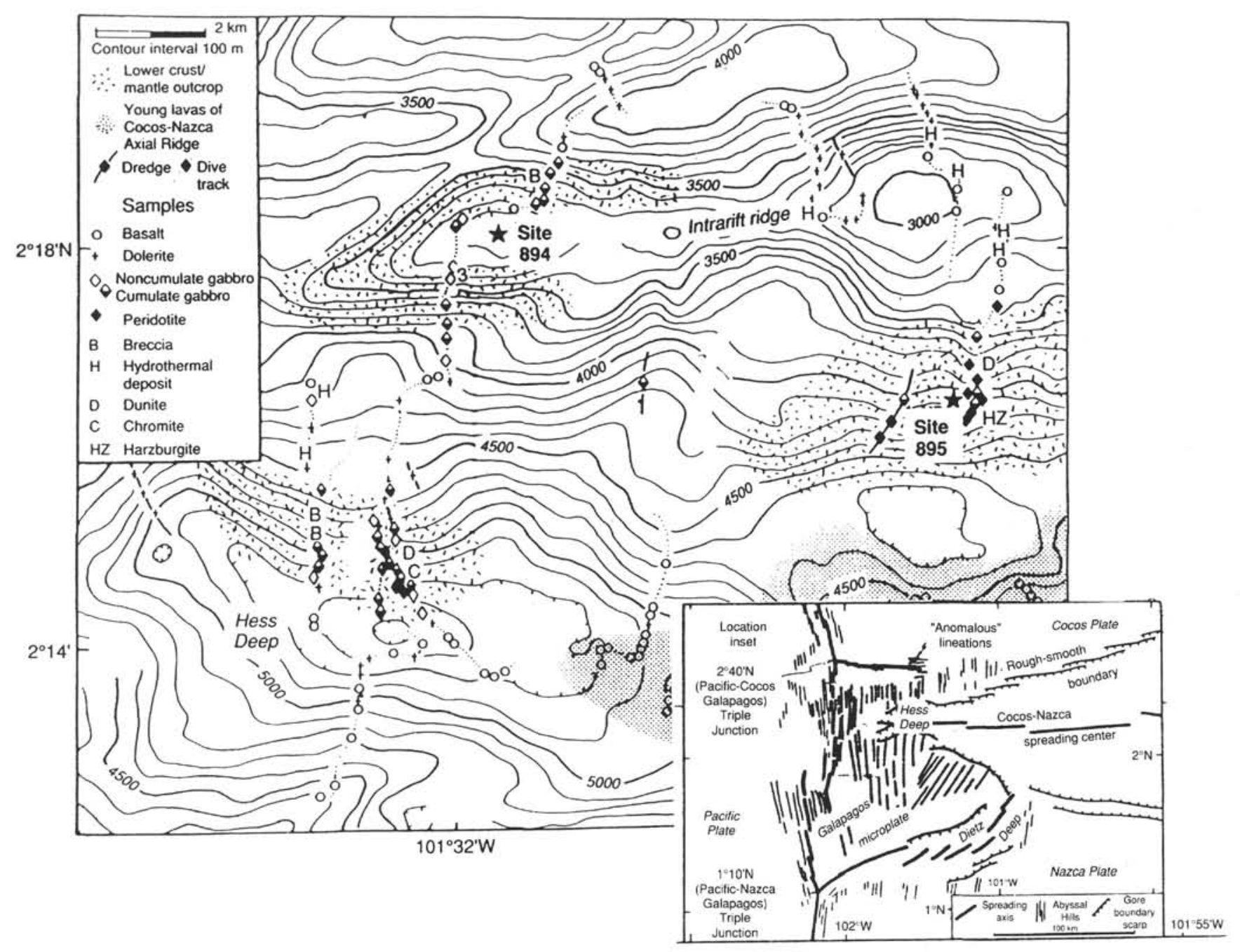

Figure 1. Location map of Hess Deep sites cored during Leg 147 (from Gillis, Mével, Allan, et al., 1993). The geology shown is modified from Francheteau et al. (1990).

\section{PETROGRAPHY}

For all sample descriptions, ODP conventions (Gillis, Mével, Allan, et al., 1993) are followed concerning sample identification (leg, core, core type, section, interval) and alteration terminology (fresh, $0 \%-2 \%$; slight, $2 \%-10 \%$; moderate, $10 \%-40 \%$; high, $40 \%-80 \%$; pervasive, $>80 \%$ ). Extensive petrographic descriptions of these samples are given in Gillis, Mével, Allan, et al. (1993), some of which are the actual thin sections analyzed here, so only a brief summry of the sample petrography will be given.

Harzburgites recovered at Site 895 are typical of more depleted, clinopyroxene-poor, residual harzburgites (Dick, 1989; Dick et al., 1984; Dick and Fisher, 1984), with an original protogranular texture that has been overprinted with porphyroclastic textures and other subsequent textures associated with brittle deformation and hydrothermal alteration. They contain $10 \%-20 \%$ porphyroclasts of orthopyroxene (to about $5 \mathrm{~mm}$ ), partially altered to talc, tremolite, and bastite (Früh-Green, Plas, and Lecuyer, this volume; Mével and Stamoudi, this volume), and only minor amounts (typically $<1 \%$ ) of small $(<1 \mathrm{~mm})$, anhedral clinopyroxene, commonly attached to spinel. Individual olivine grains are up to $1 \mathrm{~cm}$ in size, although they are commonly represented by relict "eyes" surrounded by serpentine alteration. Dark, reddish-brown spinel (to about $5 \mathrm{~mm}$ ) occurs in a variety of morphologies, ranging from blocky anhedral (PI. IA) to anhedral "holly-leaf" shapes (PI. 1B), with vermicular forms less common but present. Although much fresher than typical abyssal peridotites and fresher than the harzburgites from Hole $895 \mathrm{E}$, the analyzed harzburgites from Holes $895 \mathrm{~A}, 895 \mathrm{C}$, and 895D still carry about $50 \%-80 \%$ alteration minerals.

The original protogranular texture of the harzburgites is interpreted to be associated with asthenospheric flow; the porphyroclastic textures are interpreted to be associated with later, subsolidus flow, whereas the hydrothermal and brittle deformation textures are thought to be related to the Hess Deep opening (Boudier et al., this volume; Dick and Natland, this volume; Kennedy et al., this volume). The major and trace element mineral compositions of the harzburgites lie at the extreme depleted end of the field for residual harzburgites worldwide (Dick and Natland, this volume), but do not extend to the extremely depleted compositions found in many ophiolitic peridotites (Dick and Fisher, 1984).

Dunite samples from Site 895 contain little or no fresh pyroxene, and are notable in that they are substantially more altered than the associated harzburgites, exhibiting extensive to pervasive alteration of their olivine to serpentine. Bastite pseudomorphs of orthopyroxene are present in small amounts $(0 \%-10 \%$; Gillis, Mével, Allan, et al., 1993). Spinel within the analyzed dunites is dark reddish-brown to 

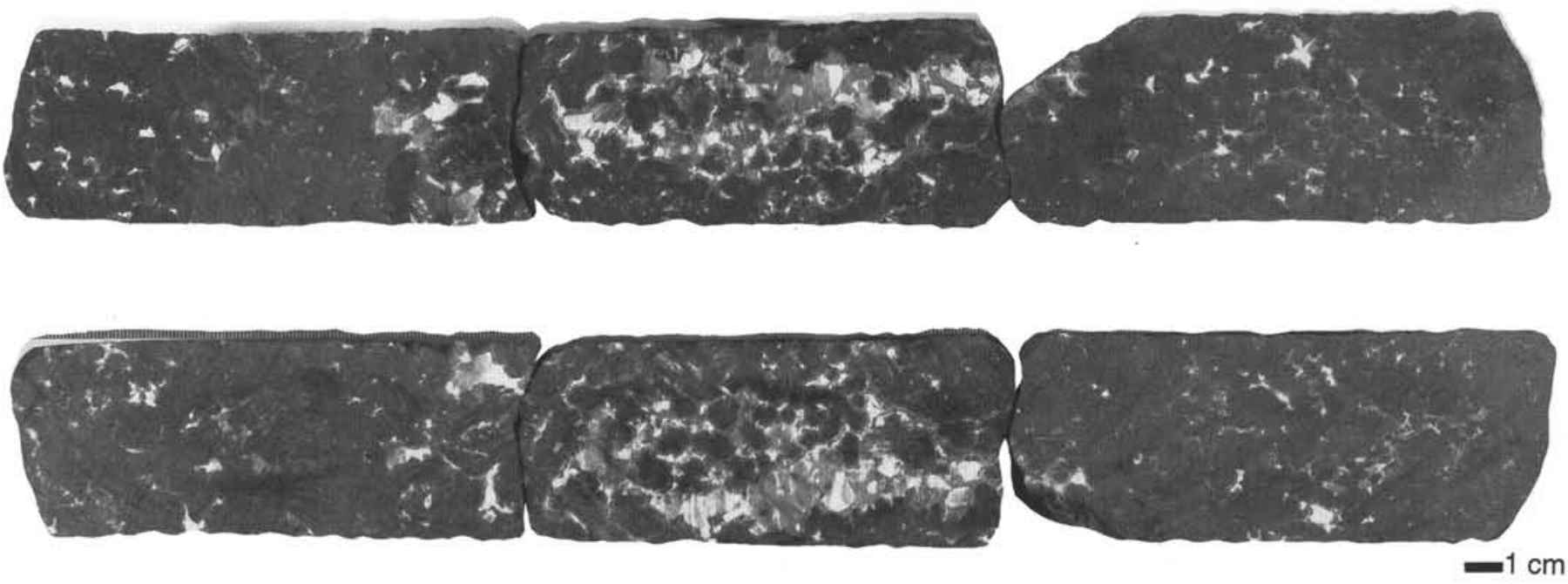

Figure 2. Photograph of core showing pervasive melt impregnation into peridotite. Harzburgite occurring at the top of Core 147-895C-4R grades into orthopyroxene-poor dunite, which in turn is intimately invaded by intergranular gabbroic rock. Near and within the dunite, visible at the top of the photograph, are interstitial, irregular patches of anhedral plagioclase, clinopyroxene, olivine, and $\mathrm{Cr}$-spinel that increase in size and abundance down the core to the center of the photograph. The melt patches are overprinted with lower amphibolite to zeolite facies metamorphic minerals. Troctolite samples analyzed in this paper come from the margins of the interval shown here.

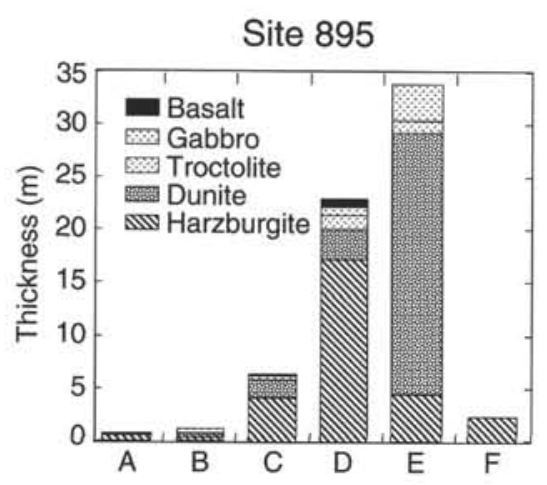

Figure 3. Comparative rock recoveries from the six Site 895 holes, shown in the recorded thickness of the recovered rocks. Note the relative abundance of both gabbroic rocks and dunite at Hole 895E.

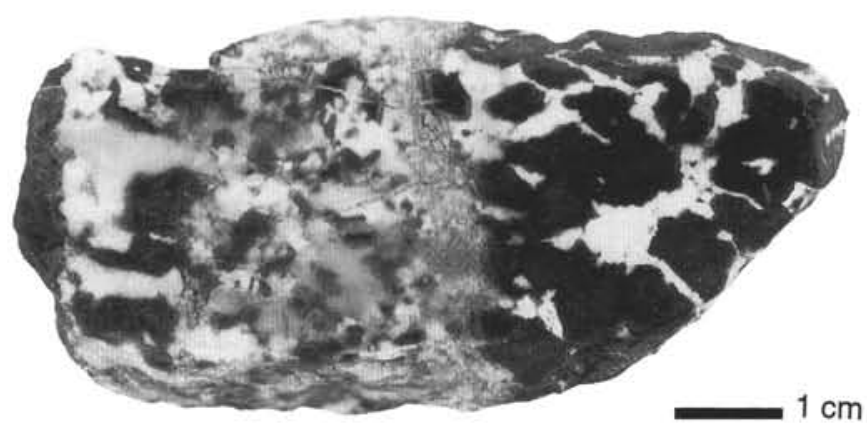

Figure 4. A rare contact recovered in core between troctolite and an apparently later gabbroic intrusion. Note the chilled margin on the gabbro. black, and is typically more rounded in shape than those in the harzburgites, with anhedral, holly-leaf textures less well developed (PI. 1C, D). Spinel grains commonly occur in elongate trains, with these trains also occurring in harzburgite and most likely representing a foliation fabric (Gillis, Mével, Allan, et al., 1993).

Troctolites from Hole $895 \mathrm{C}$ are named on a modal basis and represent an intimate patchwork of host dunite pods and impregnating gabbroic veins and patches (Fig. 3). Typically, the ratio of gabbroic rock to dunitic pods is less than 1 ; these samples could alternatively be called plagioclase dunites, depending on the amount of plagioclase present. The gaobroic segregations consist predominantly of anhedral plagioclase and clinopyroxene that have been heavily altered to secondary amphibolite to zeolite minerals (Gillis, Mével, Allan, et al., 1993; Früh-Green, Plas, and Lecuyer, this volume; Mével and Stamoudi, this volume). They are typically irregular in shape, with their edges defined by the heavily altered, surrounding olivine kernels (Pl. 1E). Dark, nearly opaque spinel is abundant (to 5\% of the samples analyzed here), and occurs in two forms: blocky, subhedral grains to several millimeters in size (PI. $1 \mathrm{~F})$, and large (to $1 \mathrm{~cm}$ ), skeletal grains that typically include plagioclase and unexsolved clinopyroxene (PI. 1F). These spinel grains may occur as crystals within or on the margins of gabbroic patches (Pl. $\mathrm{IE})$, or they may occur in isolation, surrounded by olivine (Pl. IF).

Olivine gabbro in the samples analyzed contains a much lower percentage of olivine. Where fresh (such as in Sample 147-895D-7R$2,71-75 \mathrm{~cm}$; Pl. 1G), olivine occurs in isolated clusters of millimetersized grains, surrounded by granular and intergranular clinopyroxene and plagioclase that is partially to completely altered to amphibolite to zeolite metamorphic minerals (Gillis, Mével, Allan, et al., 1993; Früh-Green, Plas, and Lecuyer, this volume; Mével and Stamoudi, this volume). Spinel is dark and nearly opaque, occurring as small $(0.05-0.4 \mathrm{~mm})$, commonly rounded, subhedral to euhedral grains or inclusions in olivine and plagioclase (PI. $1 \mathrm{G}, \mathrm{H})$. Other gabbroic samples lack fresh olivine and usually occur farther away from dunite in recovered core; these are typically very highly to pervasively altered to secondary minerals, with spinel occurring as small $(<0.3 \mathrm{~mm})$, surprisingly fresh, isolated euhedra. Spinel abundance in these samples (Samples 147-895D-10W-1, 82-85 cm, 147-895D-8R-2, 42-44 cm, 
and 147-895E-1R-1, 109-112 cm, analyzed here) is much lower than in the other, more olivine-rich gabbro samples.

\section{ANALYTICAL METHODS}

A major goal of this study was to measure accurately a variety of minor elements (including $\mathrm{Ti}, \mathrm{V}, \mathrm{Zn}, \mathrm{Co}$, and $\mathrm{Sc}$ ) in the spinels to use as indicators of mass transfer processes. An additional goal was to make high-quality estimations of spinel $\mathrm{Fe}_{2} \mathrm{O}_{3}$ and $\mathrm{Fe}^{3+} /(\mathrm{Cr}+\mathrm{Al}+$ $\mathrm{Fe}^{3+}$ ) (Carmichael, 1967), estimations that require complete elemental analysis in order to be successful. Electron microprobe analyses of spinel were obtained on a Cameca SX-50 microprobe using Smithsonian natural mineral standards (Jarosowich et al., 1980) and pure metal standards, a focused beam, and a sample current of $30 \mathrm{nA}$. Great care was taken to avoid overt alteration in the spinel grains, with back-scattered electron images taken of each grain before the analysis location was planned (Fig. 5). Unusually long counting times (60-180 s/element) were utilized for all elements except for Fe, $\mathrm{Ca}$, and $\mathrm{Si}$, which were counted for 30,30 , and $10 \mathrm{~s}$, respectively. Special care was also taken in placing where peak and background counts were taken in order to avoid peak overlap problems. Nevertheless, peak overlap corrections, using interference counts made on highly purified metal and rutile, were made for correction of interference of $\mathrm{Cr}$ on $\mathrm{Mn}, \mathrm{Ti}$ on $\mathrm{V}$, and $\mathrm{Fe}$ on $\mathrm{Co}$. Unfortunately, the analytic procedure employed was not able to measure $\mathrm{Co}$ and $\mathrm{Sc}$ reliably, for which these oxides are estimated at $\leq 0.02 \%$ abundance in the Hess Deep spinels. Results of these analyses are presented in Tables 1 and 2 and Figures 6 through 8 . It is important to note that $\mathrm{Cr} \#$ in this paper relates to $\mathrm{Cr} /\left(\mathrm{Cr}+\mathrm{Al}+\mathrm{Fe}^{3+}\right)$ rather than $\mathrm{Cr} /(\mathrm{Cr}+\mathrm{Al})$ in order to note the composition of $\mathrm{Cr}$ relative to all of the major trivalent spinel cations. Selected grains of coexisting olivine, pyroxene, and plagioclase were also analyzed on the electron microprobe, using a focused beam, sample currents of $10-30 \mathrm{nA}$, counting times of 10 to $60 \mathrm{~s}$, and both natural mineral (Jarosowich et al., 1980) and metal standards. These analyses are presented in Tables 3 and 4.

\section{SPINEL CHEMISTRY}

\section{Spinel Metamorphic Overprint}

The Leg 147 Hess Deep peridotites are composite rocks, originating from a combination of igneous processes and high-temperature metamorphic processes in the upper mantle. They are progressively overprinted with the effects of (1) high-temperature $\left(>500^{\circ} \mathrm{C}\right)$, nearridge amphibolite facies metamorphism; (2) dominant, lower temperature $\left(350-450^{\circ} \mathrm{C}\right)$, off-ridge greenschist facies metamorphism that resulted in extensive serpentinization; and (3) subsequent low-temperature zeolite alteration (Früh-Green, Plas, and Lecuyer, this volume; Mével and Stamoudi, this volume; Gillis, Mével, Allan, et al., 1993). In addition, some amounts of $\mathrm{Mg}-\mathrm{Fe}^{2+}$ exchange between spinel and olivine during peridotite cooling before amphibolite-zeolite metamorphism has occurred (e.g., Ozawa, 1983). As a result, interpretation of spinel phase chemistry, including the understanding of basic elemental variation and covariation, is difficult. Our principal interest is to examine variations in spinel composition that result from processes associated with melt impregnation and melt-wall rock interaction, which is a difficult task without removal of the effects of the lower temperature metamorphic overprint.

Each analyzed spinel was imaged by back-scattered electrons in order to avoid analyzing regions of obvious ferritchromite formation that commonly occur along grain boundaries and crystal fractures (Fig. 5). Despite this precaution, examination of the electron microprobe analytical transects shows that many of the analyses of spinel reflect compositional effects of metamorphic exchange after initial crystallization, as exemplified by slight to sharp lowering in $\mathrm{Mg} /(\mathrm{Mg}$



Figure 5. Back-scattered electron image of a spinel from Sample 147-895E$2 \mathrm{R}-1,36-39 \mathrm{~cm}$. This is the same grain shown by analytical transect A1 in Figure 6; it is also shown in Plate $1 \mathrm{H}$.

$\left.+\mathrm{Fe}^{2+}\right)(\mathrm{Mg} \#)$, which is typically accompanied by a loss in $\mathrm{Ti}$ at the grain margins (Fig. 6). Severe lowering in $\mathrm{Mg \#}$ at spinel margins, caused by $\mathrm{Mg}$ loss and $\mathrm{Fe}^{2+}$ gain (such as in the electron microprobe transects of Sample 147-895E-2R-1, 36-39 cm, shown in Fig. 6 and by the associated analyses A1-IPC, A1-IPR, B1-IPC, and B1-IPR in Table 2), is usually accompanied by increases in $\mathrm{ZnO}$ and $\mathrm{MnO}$. Similar increases in $\mathrm{MnO}$ occur in spinel from alpine peridotites affected by low-temperature alteration (S. Arai, pers. comm., 1994); the increase in $\mathrm{ZnO}$ (up to $0.75 \%$ ) probably reflects exchange with $\mathrm{Zn}$-rich hydrothermal fluids.

A key compositional feature of this metamorphic overprint is the lack of any significant change in $\mathrm{Cr} \#$, even with a greater than fourfold decrease in $\mathrm{Mg} \#$. This cannot possibly represent an igneous process related to exchange with an evolving melt, owing to the strong compositional coupling between $\mathrm{Fe}^{2+}$ and $\mathrm{Cr}^{3+}$ and $\mathrm{Mg}^{2+}$ and $\mathrm{Al}^{3+}$ in the spinel structure (Sack, 1982; Sack and Ghiorso, 1991a, 1991b). This is illustrated by examining the free energy of exchange $\left(\Delta \mathrm{G}_{\mathrm{EX}}\right)$ for the appropriate reactions:

$$
\begin{gathered}
\mathrm{MgCr}_{2} \mathrm{O}_{4}+\mathrm{FeAl}_{2} \mathrm{O}_{4} \leftrightarrow \mathrm{MgAl}_{2} \mathrm{O}_{4}+\mathrm{FeCr}_{2} \mathrm{O}_{4} \\
\mathrm{G}_{\mathrm{EX}} \approx-23 \mathrm{~kJ} \mathrm{Mol}^{-1} \text { (Sack and Ghiorso, 1991a, 1991b) }
\end{gathered}
$$

in the solid spinel phase, and

$$
\begin{gathered}
\left(\mathrm{Mg}^{2+}\right)_{\text {Melt }}+\left(\mathrm{Fe}^{2+}\right)_{\text {Spinel }} \leftrightarrow\left(\mathrm{Mg}^{2+}\right)_{\text {Spinel }}+\left(\mathrm{Fe}^{2+}\right)_{\text {Melt }} \\
\Delta \mathrm{G}_{\mathrm{EX}} \approx-13 \mathrm{~kJ} \mathrm{Mol}^{-1} \text { (Allan et al., 1988; Allan, 1992). }
\end{gathered}
$$

These relations demand that during equilibrium exchange between spinel and melt, large drops in Mg\# must be accompanied by 
Table 1. Summary of Cr-spinel electron microprobe analyses.

\begin{tabular}{|c|c|c|c|c|c|c|c|c|c|c|c|c|c|c|c|}
\hline $\begin{array}{l}\text { Core, section, } \\
\text { interval }(\mathrm{cm})\end{array}$ & Rock type & $\begin{array}{l}\text { Depth } \\
\text { (mbsf) }\end{array}$ & Unit & $\mathrm{TiO}_{2}$ & $\mathrm{~V}_{2} \mathrm{O}_{3}$ & $\mathrm{MnO}$ & $\mathrm{NiO}$ & $\mathrm{ZnO}$ & $\mathrm{Fe}^{3+\#}$ & Cr\# & Mg\# & $\begin{array}{l}\text { Cr-spinel } \\
\text { anal. \# }\end{array}$ & $\begin{array}{l}\text { Olivine } \\
\text { Fo }\end{array}$ & $\begin{array}{l}\text { Olivine } \\
\text { anal.\# }\end{array}$ & $\begin{array}{l}\text { Olivine-spinel } \\
\text { temperature }\left({ }^{\circ} \mathrm{C}\right)\end{array}$ \\
\hline \multicolumn{16}{|l|}{$\begin{array}{l}\text { All analyses } \\
\text { 147-895D- }\end{array}$} \\
\hline $10 \mathrm{~W}-1,82-85$ & Gabbro & $0-93.7$ & None & $0.81-1.04$ & $0.27-0.32$ & $0.24-0.39$ & $0.07-0.15$ & $0.13-0.20$ & $0.05-0.09$ & $0.51-0.58$ & $0.42-0.51$ & 10 & & & \\
\hline $7 \mathrm{R}-2,71-75$ & Olivine gabbro & 66.8 & 18 & $0.92-2.56$ & $0.16-0.65$ & $0.26-0.62$ & $0.04-0.15$ & $0.07-0.38$ & $0.09-0.15$ & $0.35-0.54$ & $0.25-0.50$ & 45 & & & \\
\hline $8 \mathrm{R}-2,42-44$ & Olivine gabbro & 76.1 & 32 & $0.52-1.21$ & $0.20-0.34$ & $0.17-0.47$ & $0.09-0.15$ & $0.06-0.21$ & $0.07-0.08$ & $0.50-0.53$ & $0.32-0.61$ & 24 & & & \\
\hline \multicolumn{16}{|l|}{ 147-895E- } \\
\hline $\begin{array}{l}\text { IR-1, 109-112 } \\
2 \mathrm{R}-1,36-39\end{array}$ & $\begin{array}{l}\text { Olivine gabbro } \\
\text { Olivine gabbro }\end{array}$ & 1.1 & 2 & $0.71-1.16$ & $0.18-0.38$ & $0.23-0.30$ & $0.09-0.15$ & $0.08-0.15$ & $0.04-0.07$ & $0.44-0.52$ & $0.43-0.49$ & 8 & & & \\
\hline 2R-1,36-39 & Olivine gabbro & 20.0 & 10 & $0.92-1.77$ & $0.32-1.01$ & $0.24-1.11$ & $0.05-0.14$ & $0.04-0.75$ & $0.05-0.12$ & $0.45-0.60$ & $0.13-0.58$ & 12 & & & \\
\hline \multicolumn{16}{|c|}{ 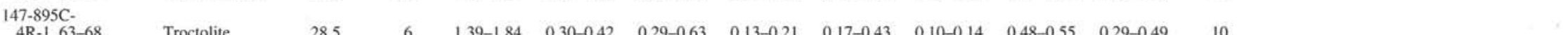 } \\
\hline $4 \mathrm{R}-2,75-78$ & Troctolite & 30.2 & 6 & $0.84-1.52$ & $0.26-0.32$ & $0.23-0.38$ & $0.13-0.20$ & $0.08-0.22$ & $0.07-0.11$ & $0.49-0.55$ & $0.40-0.56$ & 41 & & & \\
\hline $4 \mathrm{R}-2,126-128$ & Troctolite & 30.7 & 6 & $0.72-1.14$ & $0.23-0.30$ & $0.26-0.35$ & $0.13-0.20$ & $0.08-0.19$ & $0.07-0.09$ & $0.46-0.53$ & $0.51-0.60$ & 20 & & & \\
\hline \multicolumn{16}{|c|}{$\begin{array}{cccccccc}0.44-0.48 & 0.17-0.23 & 0.23-0.33 & 0.11-0.17 & 0.08-0.24 & 0.05-0.07 & 0.47-0.50 & 0.50-0.61\end{array}$} \\
\hline $9 \mathrm{R}-1,101-105$ & Dunite & 85.0 & 42 & $0.44-0.48$ & $0.17-0.23$ & $0.23-0.33$ & $0.11-0.17$ & $0.08-0.24$ & $0.05-0.07$ & $0.47-0.50$ & $0.50-0.61$ & 20 & & & \\
\hline \multicolumn{2}{|c|}{$0.90-1.10 \quad 0.21-0.28 \quad 0.14-0.26 \quad 0.12-0.19 \quad 0.08-0.20 \quad 0.04-0.06 \quad 0.51-0.53 \quad 0.57-0.62$} & 62.4 & 43 & $0.90-1.10$ & $0.21-0.28$ & $0.14-0.26$ & $0.12-0.19$ & $0.08-0.20$ & $0.04-0.06$ & $0.51-0.53$ & $0.57-0.62$ & 34 & & & \\
\hline \multicolumn{15}{|c|}{ 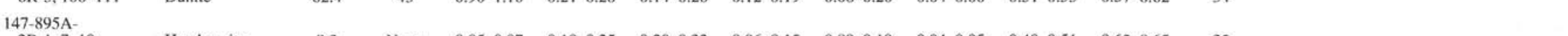 } & \\
\hline \multicolumn{16}{|c|}{$0.05-0.07 \quad 0.19-0.25 \quad 0.22-0.32 \quad 0.06-0.14 \quad 0.09-0.20$} \\
\hline $4 \mathrm{R}-1,10$ & Harzburgite & 30.0 & 5 & $0.05-0.07$ & $0.19-0.25$ & $0.22-0.32$ & $0.06-0.14$ & $0.09-0.20$ & 0.05 & $0.51-0.53$ & $0.50-0.58$ & 16 & & & \\
\hline \multicolumn{16}{|l|}{ 147-895D- } \\
\hline $5 \mathrm{R}-1,83-86$ & Harzburgite & 51.1 & 9 & $0.03-0.07$ & $0.18-0.24$ & $0.15-0.31$ & $\begin{array}{l}0.09-0.10 \\
0.05-0.15\end{array}$ & $\begin{array}{l}0.22-0.23 \\
0.08-0.20\end{array}$ & $\begin{array}{l}0.03-0.04 \\
0.03-0.04\end{array}$ & $\begin{array}{c}0.52 \\
0.51-0.53\end{array}$ & $\begin{array}{l}0.60-0.61 \\
0.62-0.66\end{array}$ & 34 & & & \\
\hline $7 \mathrm{R}-2,123-126$ & Harzburgite & 67.3 & 19 & $0.01-0.06$ & $0.19-0.27$ & $0.21-0.32$ & $0.05-0.15$ & $0.09-0.23$ & $0.02-0.04$ & $0.52-0.56$ & $0.52-0.62$ & 23 & & & \\
\hline \multicolumn{16}{|l|}{$\begin{array}{l}\text { Unzoned cores } \\
\text { 147-895D- }\end{array}$} \\
\hline $10 \mathrm{~W}-1,82-85$ & Gabbro & $0-93.7$ & None & $0.85-0.96$ & $0.27-0.32$ & $0.25-0.30$ & $0.08-0.13$ & $0.13-0.19$ & $0.05-0.08$ & $0.56-0.57$ & $0.50-0.51$ & 8 & * & & \\
\hline $7 \mathrm{R}-2,71-75$ & Olivine gabbro & 66.8 & 18 & $0.92-2.56$ & $0.16-0.65$ & $0.26-0.45$ & $0.04-0.15$ & $0.07-0.36$ & $0.10-0.13$ & $0.35-0.54$ & $0.30-0.50$ & 24 & $83-84$ & 12 & $770-830$ \\
\hline $8 R-2,42-44$ & Olivine gabbro & 76.1 & 32 & $0.55-1.21$ & $0.19-0.36$ & $0.16-0.27$ & $0.09-0.15$ & $0.06-0.20$ & $0.07-0.08$ & $0.50-0.52$ & $0.50-0.60$ & 21 & * & & \\
\hline \multicolumn{16}{|l|}{ 147-895E- } \\
\hline $\begin{array}{l}\text { RR-1, } 199-112 \\
2 R-1,36-39\end{array}$ & $\begin{array}{l}\text { Olivine gabbro } \\
\text { Olivine gabbro }\end{array}$ & $\begin{array}{c}1.1 \\
20.0\end{array}$ & 10 & $\begin{array}{l}0.84-1.10 \\
1.34-1.77\end{array}$ & $0.37-0.38$ & $\begin{array}{l}0.24-0.30 \\
0.25-0.26\end{array}$ & $\begin{array}{l}0.11-0.15 \\
0.09-0.13\end{array}$ & $\begin{array}{l}0.08-0.15 \\
0.04-0.12\end{array}$ & $\begin{array}{l}0.06-0.07 \\
0.06-0.07\end{array}$ & $\begin{array}{l}0.46-0.51 \\
0.52-0.53\end{array}$ & $\begin{array}{l}0.45-0.49 \\
0.57-0.58\end{array}$ & $\begin{array}{l}5 \\
4\end{array}$ & 88 & 5 & $600-640$ \\
\hline \multicolumn{16}{|l|}{$147-895 \mathrm{C}-$} \\
\hline $4 \mathrm{R}-1,63-68$ & Troctolite & 28.5 & 6 & $1.39-1.84$ & $0.30-0.42$ & $0.29-0.41$ & $0.15-0.21$ & $0.15-0.25$ & $0.10-0.14$ & $0.48-0.53$ & $0.42-0.49$ & 8 & $88-90$ & 12 & $760-820$ \\
\hline $4 \mathrm{R}-2,75-78$ & Troctolite & 30.2 & 6 & $0.94-1.52$ & $0.25-0.32$ & $0.23-0.33$ & $0.13-0.20$ & $0.07-0.22$ & $0.09-0.11$ & $0.51-0.55$ & $0.46-0.56$ & 34 & 89-90 & 7 & $800-890$ \\
\hline $4 \mathrm{R}-2,126-128$ & Troctolite & 30.7 & 6 & $0.72-1.14$ & $0.23-0.30$ & $0.20-0.35$ & $0.13-0.20$ & $0.08-0.19$ & $0.07-0.09$ & $0.46-0.52$ & $0.51-0.60$ & 20 & $89-90$ & 5 & $840-900$ \\
\hline 147-895D- & Dunite & 85.0 & 42 & $0.46-0.47$ & $0.20-0.23$ & $0.23-0.33$ & $0.11-0.17$ & $0.09-0.21$ & $0.05-0.06$ & $0.48-0.49$ & $0.59-0.61$ & 11 & ${ }^{*}$ & & \\
\hline $\begin{array}{l}147-895 \mathrm{E}- \\
6 \mathrm{R}-3,108-111\end{array}$ & Dunite & 62.4 & 43 & $0.91-1.11$ & $0.21-0.28$ & $0.14-0.26$ & $0.12-0.19$ & $0.08-0.20$ & $0.04-0.06$ & $0.50-0.53$ & $0.59-0.62$ & 33 & 90 & 5 & $860-880$ \\
\hline \multicolumn{6}{|l|}{ 147-895A- } & $0.25-0.32$ & $0.06-0.15$ & $0.09-0.16$ & $0.04-0.05$ & & $0.62-0.65$ & 24 & 91 & & \\
\hline \multicolumn{16}{|l|}{$147-895 \mathrm{C}-$} \\
\hline $4 R-1,10-12$ & Harzburgite & 30.0 & 5 & $0.05-0.07$ & $0.22-0.27$ & $0.26-0.32$ & $0.08-0.14$ & $0.11-0.21$ & 0.050 & $0.52-0.53$ & $0.56-59$ & 9 & 90-91 & 4 & 760 \\
\hline \multicolumn{16}{|l|}{ 147-895D- } \\
\hline $\begin{array}{l}4 \mathrm{R}-1,34-37 \\
5 \mathrm{R}-1,83-86\end{array}$ & $\begin{array}{l}\text { Harzburgite } \\
\text { Harzburgite }\end{array}$ & $\begin{array}{l}40.0 \\
51.1\end{array}$ & $\begin{array}{l}5 \\
9\end{array}$ & $\begin{array}{c}0.03 \\
0.03-0.07\end{array}$ & $\begin{array}{l}0.24-0.27 \\
0.19-0.24\end{array}$ & $\begin{array}{l}0.22-0.29 \\
0.22-0.31\end{array}$ & $\begin{array}{l}0.09-0.10 \\
0.06-0.15\end{array}$ & $\begin{array}{l}0.22-0.23 \\
0.09-0.20\end{array}$ & $\begin{array}{c}0.030 \\
0.03-0.04\end{array}$ & $\begin{array}{c}0.52 \\
0.51-0.52\end{array}$ & $\begin{array}{l}0.60-0.61 \\
0.62-0.66\end{array}$ & $\begin{array}{r}2 \\
27\end{array}$ & $\begin{array}{l}91 \\
91-92\end{array}$ & $\begin{array}{r}14 \\
9\end{array}$ & $\begin{array}{l}760-770 \\
740-850\end{array}$ \\
\hline $7 \mathrm{R}-2,123-126$ & Harzburgite & 67.3 & 19 & $0.02-0.06$ & $0.19-0.27$ & $0.21-0.31$ & $0.08-0.15$ & $0.09-0.23$ & $0.03-0.04$ & $0.52-0.54$ & $0.58-0.62$ & 20 & 91 & 12 & $790-820$ \\
\hline
\end{tabular}

Notes: The summary of unzoned cores shows the composition of "fresh" spinel, with analyses removed that show evidence of post-crystallization metamorphic exchange $\left(\right.$ see text). $\mathrm{Mg} \#=\mathrm{Mg} /\left(\mathrm{Mg}+\mathrm{Fe}{ }^{3+}\right), \mathrm{Cr} \#=\mathrm{Cr} /\left(\mathrm{Cr}+\mathrm{Al}+\mathrm{Fe}{ }^{4+}\right)$, and $\mathrm{Fe}{ }^{3+}+\#$ $=\mathrm{Fe}^{2} /\left(\mathrm{Cr}+\mathrm{Al}+\mathrm{Fe}^{3+}\right) . \mathrm{Fo}=$ forsterite content. Olivine-spinel $(\mathrm{Ol}-\mathrm{Sp})$ temperatures were calculated using a program provided by $\mathrm{R}$. Sack, based upon the spinel solution model of Sack and $\mathrm{Ghiorso}(199 \mathrm{la}, 1991 \mathrm{~b}),{ }^{*}=$ no fresh olivine was found to analyze. 


\begin{tabular}{|c|c|c|c|c|c|c|c|c|c|c|c|c|c|c|c|c|c|c|c|c|}
\hline $\begin{array}{l}\text { Rock: } \\
\text { Hole: } \\
\text { Core, section: } \\
\text { Interval (cm): } \\
\text { Analysis \#: }\end{array}$ & $\begin{array}{c}\mathrm{G} \\
895 \mathrm{D} \\
10 \mathrm{~W}-1 \\
82-85 \\
\mathrm{~B} 1-\mathrm{C}\end{array}$ & $\begin{array}{c}\text { G } \\
895 \mathrm{D} \\
10 \mathrm{~W}-1 \\
82-85 \\
\mathrm{~A} 1-\mathrm{C}\end{array}$ & $\begin{array}{c}\mathrm{OG} \\
895 \mathrm{D} \\
7 \mathrm{R}-2 \\
71-75 \\
\mathrm{~A} 1-\mathrm{C}\end{array}$ & $\begin{array}{c}\text { OG } \\
895 \mathrm{D} \\
7 \mathrm{R}-2 \\
71-75 \\
\mathrm{~A} 1-\mathrm{R}\end{array}$ & $\begin{array}{c}\mathrm{OG} \\
895 \mathrm{D} \\
7 \mathrm{R}-2 \\
71-75 \\
4-1 \mathrm{IOC}\end{array}$ & $\begin{array}{c}\text { OG } \\
895 \mathrm{D} \\
7 \mathrm{R}-2 \\
71-75 \\
5-\mathrm{IOR}\end{array}$ & $\begin{array}{c}\text { OG } \\
895 \mathrm{D} \\
7 \mathrm{R}-2 \\
71-75 \\
11-\mathrm{IOC}\end{array}$ & $\begin{array}{c}\mathrm{OG} \\
895 \mathrm{D} \\
7 \mathrm{R}-2 \\
71-75 \\
16-\mathrm{C}\end{array}$ & $\begin{array}{c}\text { OG } \\
895 \mathrm{D} \\
7 \mathrm{R}-2 \\
71-75 \\
\text { D01-IPC }\end{array}$ & $\begin{array}{c}\mathrm{OG} \\
895 \mathrm{D} \\
7 \mathrm{R}-2 \\
71-75 \\
28-\mathrm{IPC}\end{array}$ & $\begin{array}{c}\text { OG } \\
895 \mathrm{D} \\
7 \mathrm{R}-2 \\
71-75 \\
30-\mathrm{IPC}\end{array}$ & $\begin{array}{c}\mathrm{OG} \\
895 \mathrm{D} \\
7 \mathrm{R}-2 \\
71-75 \\
33-\mathrm{C}\end{array}$ & $\begin{array}{c}\text { OG } \\
895 \mathrm{D} \\
8 \mathrm{R}-2 \\
42-44 \\
\mathrm{Al}-\mathrm{C}\end{array}$ & $\begin{array}{c}\text { OG } \\
895 \mathrm{D} \\
8 \mathrm{R}-2 \\
42-44 \\
\mathrm{~B} 1-\mathrm{C}\end{array}$ & $\begin{array}{c}\text { OG } \\
895 \mathrm{D} \\
8 \mathrm{R}-2 \\
42-44 \\
\mathrm{~B} 1-\mathrm{R}\end{array}$ & $\begin{array}{l}\mathrm{OG} \\
895 \mathrm{D} \\
8 \mathrm{R}-2 \\
42-44 \\
\mathrm{DI}-\mathrm{C}\end{array}$ & $\begin{array}{c}\mathrm{OG} \\
895 \mathrm{E} \\
1 \mathrm{R}-1 \\
109-112 \\
\mathrm{~B} 1-\mathrm{C}\end{array}$ & $\begin{array}{c}\mathrm{OG} \\
895 \mathrm{E} \\
1 \mathrm{R}-1 \\
109-112 \\
\mathrm{Al}-\mathrm{C}\end{array}$ & $\begin{array}{c}\text { OG } \\
895 E \\
2 R-1 \\
36-39 \\
\text { Al-IPC }\end{array}$ & $\begin{array}{r}\mathrm{OG} \\
895 \mathrm{E} \\
2 \mathrm{R}-1 \\
36-39 \\
\mathrm{AI}-\mathrm{IPR}\end{array}$ \\
\hline $\begin{array}{l}\mathrm{SiO}_{2} \\
\mathrm{TiO}_{2} \\
\mathrm{Al}_{2} \mathrm{O}_{3} \\
\mathrm{~V}_{2} \mathrm{O}_{3} \\
\mathrm{Cr}_{2} \mathrm{O}_{3} \\
\mathrm{FeO} \\
\mathrm{MnO} \\
\mathrm{NiO} \\
\mathrm{ZnO} \\
\mathrm{MgO} \\
\mathrm{CaO} \\
\text { Total }\end{array}$ & $\begin{array}{r}0.00 \\
0.88 \\
19.54 \\
0.32 \\
44.47 \\
22.76 \\
0.25 \\
0.08 \\
0.16 \\
10.81 \\
0.02 \\
99.29\end{array}$ & $\begin{array}{r}0.00 \\
0.95 \\
18.74 \\
0.31 \\
43.81 \\
23.92 \\
0.28 \\
0.11 \\
0.13 \\
10.71 \\
0.00 \\
98.96\end{array}$ & $\begin{array}{r}0.00 \\
2.36 \\
18.61 \\
0.59 \\
37.97 \\
31.24 \\
0.45 \\
0.14 \\
0.25 \\
7.62 \\
0.01 \\
99.24\end{array}$ & $\begin{array}{r}0.00 \\
2.14 \\
18.12 \\
0.56 \\
38.60 \\
32.00 \\
0.49 \\
0.11 \\
0.22 \\
6.75 \\
0.05 \\
99.04\end{array}$ & $\begin{array}{r}0.01 \\
2.05 \\
18.06 \\
0.61 \\
38.15 \\
33.23 \\
0.45 \\
0.14 \\
0.38 \\
6.61 \\
0.00 \\
99.69\end{array}$ & $\begin{array}{r}0.00 \\
1.88 \\
17.98 \\
0.57 \\
37.20 \\
34.88 \\
0.55 \\
0.09 \\
0.37 \\
5.48 \\
0.00 \\
99.00\end{array}$ & $\begin{array}{r}0.00 \\
2.49 \\
18.76 \\
0.52 \\
35.45 \\
32.64 \\
0.40 \\
0.14 \\
0.15 \\
8.30 \\
0.07 \\
98.92\end{array}$ & $\begin{array}{r}0.01 \\
2.22 \\
18.13 \\
0.48 \\
36.99 \\
31.56 \\
0.40 \\
0.09 \\
0.16 \\
8.60 \\
0.00 \\
98.64\end{array}$ & $\begin{array}{r}0.00 \\
2.26 \\
18.58 \\
0.54 \\
37.37 \\
31.36 \\
0.42 \\
0.10 \\
0.26 \\
8.20 \\
0.01 \\
99.10\end{array}$ & $\begin{array}{r}0.00 \\
2.14 \\
19.16 \\
0.46 \\
38.12 \\
30.72 \\
0.38 \\
0.13 \\
0.19 \\
8.43 \\
0.02 \\
99.75\end{array}$ & $\begin{array}{r}0.00 \\
1.45 \\
20.60 \\
0.38 \\
37.19 \\
28.45 \\
0.36 \\
0.04 \\
0.17 \\
10.09 \\
0.02 \\
98.75\end{array}$ & $\begin{array}{r}0.00 \\
0.93 \\
29.58 \\
0.17 \\
29.26 \\
27.42 \\
0.28 \\
0.10 \\
0.13 \\
11.24 \\
0.01 \\
99.12\end{array}$ & $\begin{array}{r}0.00 \\
1.06 \\
22.69 \\
0.27 \\
40.59 \\
21.13 \\
0.20 \\
0.15 \\
0.11 \\
13.08 \\
0.04 \\
99.32\end{array}$ & $\begin{array}{r}0.00 \\
1.18 \\
21.37 \\
0.36 \\
39.98 \\
25.27 \\
0.24 \\
0.11 \\
0.15 \\
10.83 \\
0.00 \\
99.49\end{array}$ & $\begin{array}{r}0.00 \\
0.90 \\
20.23 \\
0.30 \\
39.95 \\
29.95 \\
0.47 \\
0.09 \\
0.21 \\
6.63 \\
0.05 \\
98.78\end{array}$ & $\begin{array}{r}0.00 \\
0.57 \\
22.72 \\
0.22 \\
40.77 \\
21.51 \\
0.19 \\
0.09 \\
0.11 \\
13.12 \\
0.05 \\
99.35\end{array}$ & $\begin{array}{r}0.01 \\
0.84 \\
24.67 \\
0.18 \\
36.91 \\
25.44 \\
0.24 \\
0.15 \\
0.12 \\
10.60 \\
0.01 \\
99.17\end{array}$ & $\begin{array}{r}0.00 \\
1.16 \\
22.21 \\
0.36 \\
39.08 \\
26.22 \\
0.24 \\
0.12 \\
0.14 \\
9.64 \\
0.01 \\
99.18\end{array}$ & $\begin{array}{r}0.00 \\
1.50 \\
21.89 \\
0.37 \\
41.30 \\
21.49 \\
0.25 \\
0.13 \\
0.12 \\
12.84 \\
0.02 \\
99.91\end{array}$ & $\begin{array}{r}0.02 \\
11.16 \\
18.93 \\
0.32 \\
40.31 \\
33.87 \\
1.11 \\
0.08 \\
0.75 \\
2.56 \\
0.10 \\
99.21\end{array}$ \\
\hline $\begin{array}{l}\mathrm{RFcO} \\
\mathrm{Fe}_{2} \mathrm{O}_{3} \\
\text { Total }\end{array}$ & $\begin{array}{r}18.76 \\
4.44 \\
99.73\end{array}$ & $\begin{array}{r}18.76 \\
5.73 \\
99.53\end{array}$ & $\begin{array}{r}24.54 \\
7.45 \\
99.99\end{array}$ & $\begin{array}{r}25.50 \\
7.23 \\
99.76\end{array}$ & $\begin{array}{r}25.78 \\
8.28 \\
100.52\end{array}$ & $\begin{array}{r}27.08 \\
8.67 \\
99.87\end{array}$ & $\begin{array}{r}23.65 \\
9.99 \\
99.92\end{array}$ & $\begin{array}{r}22.89 \\
9.64 \\
99.60\end{array}$ & $\begin{array}{r}23.59 \\
8.63 \\
99.97\end{array}$ & $\begin{array}{r}23.48 \\
8.04 \\
100.56\end{array}$ & $\begin{array}{r}20.37 \\
8.98 \\
99.65\end{array}$ & $\begin{array}{r}19.74 \\
8.54 \\
99.98\end{array}$ & $\begin{array}{r}15.98 \\
5.72 \\
99.89\end{array}$ & $\begin{array}{r}19.41 \\
6.52 \\
100.14\end{array}$ & $\begin{array}{r}24.83 \\
5.69 \\
99.35\end{array}$ & $\begin{array}{r}15.57 \\
6.61 \\
100.01\end{array}$ & $\begin{array}{r}19.85 \\
6.21 \\
99.79\end{array}$ & $\begin{array}{r}21.21 \\
5.56 \\
99.74\end{array}$ & $\begin{array}{r}16.79 \\
5.23 \\
100.43\end{array}$ & $\begin{array}{r}30.04 \\
4.25 \\
99.64\end{array}$ \\
\hline $\begin{array}{l}\text { Fe\# } \\
\text { Cr\#\# } \\
\text { Mg\# }\end{array}$ & $\begin{array}{l}0.054 \\
0.571 \\
0.507\end{array}$ & $\begin{array}{l}0.071 \\
0.567 \\
0.504\end{array}$ & $\begin{array}{l}0.097 \\
0.521 \\
0.356\end{array}$ & $\begin{array}{l}0.095 \\
0.532 \\
0.321\end{array}$ & $\begin{array}{l}0.108 \\
0.523 \\
0.314\end{array}$ & $\begin{array}{l}0.114 \\
0.515 \\
0.265\end{array}$ & $\begin{array}{l}0.130 \\
0.486 \\
0.385\end{array}$ & $\begin{array}{l}0.125 \\
0.505 \\
0.401\end{array}$ & $\begin{array}{l}0.112 \\
0.510 \\
0.382\end{array}$ & $\begin{array}{l}0.103 \\
0.513 \\
0.390\end{array}$ & $\begin{array}{l}0.112 \\
0.486 \\
0.469\end{array}$ & $\begin{array}{l}0.100 \\
0.359 \\
0.504\end{array}$ & $\begin{array}{l}0.068 \\
0.508 \\
0.593\end{array}$ & $\begin{array}{l}0.079 \\
0.512 \\
0.499\end{array}$ & $\begin{array}{l}0.072 \\
0.529 \\
0.322\end{array}$ & $\begin{array}{l}0.078 \\
0.504 \\
0.600\end{array}$ & $\begin{array}{l}0.074 \\
0.464 \\
0.488\end{array}$ & $\begin{array}{l}0.068 \\
0.504 \\
0.447\end{array}$ & $\begin{array}{l}0.063 \\
0.523 \\
0.577\end{array}$ & $\begin{array}{l}0.056 \\
0.555 \\
0.132\end{array}$ \\
\hline
\end{tabular}

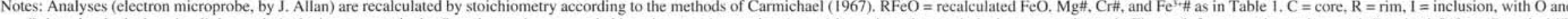
P denoting inclusions in olivine and plagioclase, respectively. Sample numbers preceded by a letter and a number (e.g., A1) are keyed to analytical transects shown in Figure 6; for example, analyses A1-C and A1-R from Sample 147895D-7R-2, 71-75 cm, refer to the core and rim analyses of transect $\mathrm{A} 1$ in the figure.

Table 2 (continued).

\begin{tabular}{|c|c|c|c|c|c|c|c|c|c|c|c|c|c|c|c|c|c|c|c|}
\hline $\begin{array}{l}\text { Rock: } \\
\text { Hole: } \\
\text { Core, section: } \\
\text { Interval (cm): } \\
\text { Analysis \#: }\end{array}$ & $\begin{array}{c}\text { OG } \\
895 \mathrm{E} \\
2 \mathrm{R}-1 \\
36-39 \\
\mathrm{BI}-\mathrm{IPC}\end{array}$ & $\begin{array}{c}\text { OG } \\
895 \mathrm{E} \\
2 \mathrm{R}-1 \\
36-39 \\
\text { B1-IPR }\end{array}$ & $\begin{array}{c}\mathrm{OG} \\
895 \mathrm{E} \\
2 \mathrm{R}-1 \\
36-39 \\
12\end{array}$ & $\begin{array}{c}\mathrm{T} \\
895 \mathrm{C} \\
4 \mathrm{R}-1 \\
63-68 \\
1-\mathrm{C}\end{array}$ & $\begin{array}{c}\mathrm{T} \\
895 \mathrm{C} \\
4 \mathrm{R}-1 \\
63-68 \\
5-\mathrm{C}\end{array}$ & $\begin{array}{c}\mathrm{T} \\
895 \mathrm{C} \\
4 \mathrm{R}-1 \\
63-68 \\
7-\mathrm{C}\end{array}$ & $\begin{array}{c}\mathrm{T} \\
895 \mathrm{C} \\
4 \mathrm{R}-1 \\
63-68 \\
8-\mathrm{C}\end{array}$ & $\begin{array}{c}\mathrm{T} \\
895 \mathrm{C} \\
4 \mathrm{R}-2 \\
75-78 \\
\mathrm{Cl}-\mathrm{C}\end{array}$ & $\begin{array}{c}\mathrm{T} \\
895 \mathrm{C} \\
4 \mathrm{R}-2 \\
75-78 \\
\mathrm{C} 2-\mathrm{C}\end{array}$ & $\begin{array}{c}\mathrm{T} \\
895 \mathrm{C} \\
4 \mathrm{R}-2 \\
75-78 \\
\mathrm{~B} 1-\mathrm{C}\end{array}$ & $\begin{array}{c}\mathrm{T} \\
895 \mathrm{C} \\
4 \mathrm{R}-2 \\
75-78 \\
\text { B2-C }\end{array}$ & $\begin{array}{c}\mathrm{T} \\
895 \mathrm{C} \\
4 \mathrm{R}-2 \\
75-78 \\
\mathrm{Al}-\mathrm{C}\end{array}$ & $\begin{array}{c}\mathrm{T} \\
895 \mathrm{C} \\
4 \mathrm{R}-2 \\
75-78 \\
\mathrm{~A} 2-\mathrm{R}\end{array}$ & $\begin{array}{c}\mathrm{T} \\
895 \mathrm{C} \\
4 \mathrm{R}-2 \\
126-128 \\
\mathrm{AAl}-\mathrm{C}\end{array}$ & $\begin{array}{c}\mathrm{T} \\
895 \mathrm{C} \\
4 \mathrm{R}-2 \\
126-128 \\
\text { BBI-C }\end{array}$ & $\begin{array}{c}\mathrm{T} \\
895 \mathrm{C} \\
4 \mathrm{R}-2 \\
126-128 \\
\text { BB2-C }\end{array}$ & $\begin{array}{c}\mathrm{D} \\
895 \mathrm{D} \\
9 \mathrm{R}-1 \\
101-105 \\
\mathrm{Al}-\mathrm{C}\end{array}$ & $\begin{array}{c}\mathrm{D} \\
895 \mathrm{D} \\
9 \mathrm{R}-1 \\
101-105 \\
\mathrm{~A} 2-\mathrm{C}\end{array}$ & $\begin{array}{c}\mathrm{D} \\
895 \mathrm{D} \\
9 \mathrm{R}-1 \\
101-105 \\
\mathrm{~A} 2-\mathrm{R}\end{array}$ \\
\hline $\begin{array}{l}\mathrm{SiO}_{2} \\
\mathrm{TiO}_{2} \\
\mathrm{Al}_{2} \mathrm{O}_{3} \\
\mathrm{~V}_{2} \mathrm{O}_{3} \\
\mathrm{Cr}_{3} \mathrm{O}_{3} \\
\mathrm{FeO} \\
\mathrm{MnO} \\
\mathrm{NiO} \\
\mathrm{ZnO} \\
\mathrm{MgO} \\
\mathrm{CaO} \\
\text { Total }\end{array}$ & $\begin{array}{r}0.06 \\
1.77 \\
21.05 \\
0.38 \\
41.26 \\
21.85 \\
0.26 \\
0.09 \\
0.10 \\
12.84 \\
0.00 \\
99.66\end{array}$ & $\begin{array}{r}0.00 \\
1.40 \\
25.47 \\
0.40 \\
34.15 \\
30.86 \\
0.72 \\
0.10 \\
0.23 \\
6.38 \\
0.03 \\
99.74\end{array}$ & $\begin{array}{r}0.00 \\
1.32 \\
14.82 \\
1.01 \\
40.18 \\
35.22 \\
0.71 \\
0.05 \\
0.25 \\
4.71 \\
0.00 \\
98.27\end{array}$ & $\begin{array}{r}0.00 \\
1.44 \\
19.19 \\
0.34 \\
40.68 \\
26.92 \\
0.32 \\
0.15 \\
0.16 \\
10.60 \\
0.00 \\
99.80\end{array}$ & $\begin{array}{r}0.00 \\
1.65 \\
18.65 \\
0.36 \\
40.00 \\
28.23 \\
0.36 \\
0.20 \\
0.24 \\
9.94 \\
0.00 \\
99.63\end{array}$ & $\begin{array}{r}0.02 \\
1.61 \\
20.00 \\
0.42 \\
38.47 \\
28.46 \\
0.32 \\
0.21 \\
0.19 \\
10.47 \\
0.00 \\
100.16\end{array}$ & $\begin{array}{r}0.00 \\
1.84 \\
16.29 \\
0.37 \\
40.93 \\
30.80 \\
0.36 \\
0.17 \\
0.15 \\
8.89 \\
0.01 \\
99.80\end{array}$ & $\begin{array}{r}0.00 \\
0.95 \\
21.58 \\
0.27 \\
40.23 \\
23.67 \\
0.24 \\
0.14 \\
0.12 \\
12.12 \\
0.01 \\
99.33\end{array}$ & $\begin{array}{r}0.05 \\
1.04 \\
20.81 \\
0.28 \\
40.45 \\
24.41 \\
0.24 \\
0.14 \\
0.12 \\
11.83 \\
0.00 \\
99.36\end{array}$ & $\begin{array}{r}0.00 \\
1.35 \\
18.17 \\
0.31 \\
41.50 \\
27.25 \\
0.33 \\
0.16 \\
0.22 \\
9.97 \\
0.01 \\
99.28\end{array}$ & $\begin{array}{r}0.00 \\
1.50 \\
18.04 \\
0.32 \\
41.44 \\
26.70 \\
0.29 \\
0.16 \\
0.10 \\
10.65 \\
0.00 \\
99.20\end{array}$ & $\begin{array}{r}0.00 \\
1.42 \\
20.17 \\
0.29 \\
40.79 \\
24.73 \\
0.28 \\
0.19 \\
0.11 \\
11.49 \\
0.01 \\
99.47\end{array}$ & $\begin{array}{r}0.00 \\
1.35 \\
22.05 \\
0.28 \\
36.99 \\
28.86 \\
0.37 \\
0.15 \\
0.17 \\
8.52 \\
0.01 \\
98.75\end{array}$ & $\begin{array}{r}0.00 \\
1.10 \\
20.57 \\
0.25 \\
40.44 \\
24.75 \\
0.35 \\
0.16 \\
0.15 \\
11.16 \\
0.01 \\
98.95\end{array}$ & $\begin{array}{r}0.00 \\
0.83 \\
22.98 \\
0.25 \\
39.96 \\
22.33 \\
0.25 \\
0.14 \\
0.12 \\
13.00 \\
0.00 \\
99.85\end{array}$ & $\begin{array}{r}0.00 \\
0.74 \\
23.75 \\
0.23 \\
39.61 \\
22.00 \\
0.27 \\
0.17 \\
0.18 \\
13.17 \\
0.00 \\
100.12\end{array}$ & $\begin{array}{r}0.00 \\
0.47 \\
25.41 \\
0.21 \\
39.55 \\
20.61 \\
0.30 \\
0.14 \\
0.16 \\
13.01 \\
0.00 \\
99.86\end{array}$ & $\begin{array}{r}0.00 \\
0.45 \\
25.66 \\
0.21 \\
40.17 \\
19.40 \\
0.28 \\
0.13 \\
0.21 \\
13.42 \\
0.00 \\
99.93\end{array}$ & $\begin{array}{r}0.00 \\
0.44 \\
24.61 \\
0.21 \\
39.96 \\
21.87 \\
0.31 \\
0.12 \\
0.19 \\
11.27 \\
0.00 \\
98.98\end{array}$ \\
\hline $\begin{array}{l}\mathrm{RFeO} \\
\mathrm{Fe}_{2} \mathrm{O}_{3} \\
\text { Total }\end{array}$ & $\begin{array}{r}16.97 \\
5.43 \\
100.20\end{array}$ & $\begin{array}{r}26.53 \\
4.81 \\
100.22\end{array}$ & $\begin{array}{r}27.02 \\
9.12 \\
99.19\end{array}$ & $\begin{array}{r}19.64 \\
8.09 \\
100.61\end{array}$ & $\begin{array}{r}20.55 \\
8.53 \\
100.49\end{array}$ & $\begin{array}{r}20.20 \\
9.18 \\
101.08\end{array}$ & $\begin{array}{r}22.13 \\
9.63 \\
100.77\end{array}$ & $\begin{array}{r}17.20 \\
7.19 \\
100.05\end{array}$ & $\begin{array}{r}17.70 \\
7.46 \\
100.11\end{array}$ & $\begin{array}{r}20.10 \\
7.94 \\
100.08\end{array}$ & $\begin{array}{r}19.32 \\
8.21 \\
100.02\end{array}$ & $\begin{array}{r}18.34 \\
7.10 \\
100.19\end{array}$ & $\begin{array}{r}22.77 \\
6.77 \\
99.43\end{array}$ & $\begin{array}{r}18.37 \\
7.10 \\
99.66\end{array}$ & $\begin{array}{r}16.13 \\
6.89 \\
100.54\end{array}$ & $\begin{array}{r}15.89 \\
6.78 \\
100.80\end{array}$ & $\begin{array}{r}16.06 \\
5.06 \\
100.37\end{array}$ & $\begin{array}{r}15.45 \\
4.39 \\
100.37\end{array}$ & $\begin{array}{r}18.25 \\
4.02 \\
99.38\end{array}$ \\
\hline $\begin{array}{l}\text { Fe\# } \\
\text { Cr\# } \\
\text { Mg\# }\end{array}$ & $\begin{array}{l}0.066 \\
0.530 \\
0.574\end{array}$ & $\begin{array}{l}0.060 \\
0.445 \\
0.300\end{array}$ & $\begin{array}{l}0.122 \\
0.566 \\
0.237\end{array}$ & $\begin{array}{l}0.100 \\
0.528 \\
0.490\end{array}$ & $\begin{array}{l}0.107 \\
0.527 \\
0.463\end{array}$ & $\begin{array}{l}0.113 \\
0.499 \\
0.480\end{array}$ & $\begin{array}{l}0.123 \\
0.550 \\
0.417\end{array}$ & $\begin{array}{l}0.086 \\
0.508 \\
0.557\end{array}$ & $\begin{array}{l}0.090 \\
0.515 \\
0.544\end{array}$ & $\begin{array}{l}0.099 \\
0.545 \\
0.469\end{array}$ & $\begin{array}{l}0.103 \\
0.544 \\
0.496\end{array}$ & $\begin{array}{l}0.087 \\
0.525 \\
0.528\end{array}$ & $\begin{array}{l}0.084 \\
0.485 \\
0.400\end{array}$ & $\begin{array}{l}0.087 \\
0.519 \\
0.520\end{array}$ & $\begin{array}{l}0.081 \\
0.495 \\
0.589\end{array}$ & $\begin{array}{l}0.079 \\
0.486 \\
0.596\end{array}$ & $\begin{array}{l}0.059 \\
0.481 \\
0.591\end{array}$ & $\begin{array}{l}0.051 \\
0.486 \\
0.608\end{array}$ & $\begin{array}{l}0.048 \\
0.496 \\
0.524\end{array}$ \\
\hline
\end{tabular}




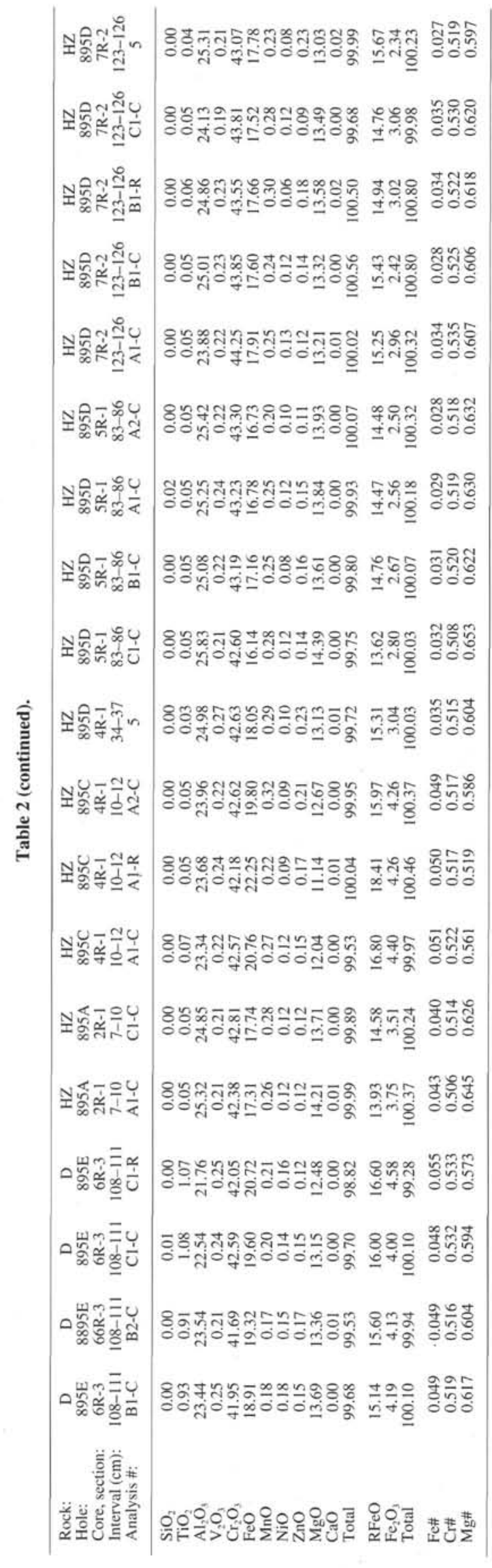

increases in Cr\# (Allan et al., 1988), as $\mathrm{Fe}^{2+}$ and $\mathrm{Cr}^{3+}$ and $\mathrm{Mg}^{2+}$ and $\mathrm{Al}^{3+}$ are energetically coupled in the spinel structure. This relationship is illustrated by the cores of spinel grains from olivine gabbro Sample 147-895D-7R-2, 71-75 cm, and troctolite Samples 147895C-4R-1,63-68 cm, 147-895C-4R-2, 75-78 cm, and 147-895C4R-2, 126-128 cm, as shown in Table 2. Similar changes in spinel chemistry, where altered grains or rims of grains show drops in Mg\# without change in $\mathrm{Cr} \#$, have been reported in a wide range of metamorphosed rocks, including both low-temperature $\left(<200^{\circ} \mathrm{C}\right)$ hydrothermally altered spinel in basalt (Allan, 1992) and in hightemperature granulite facies rocks (Sack, 1980). Engi (1983) gave numerous references that show a relatively low blocking temperature for $\mathrm{Mg}-\mathrm{Fe}^{2+}$ exchange between olivine and spinel; these studies and the Hess Deep spinel disequilibrium zoning patterns show that kinetic barriers for trivalent cation exchange are higher than for divalent cation exchange in spinel, leading to more sluggish exchange (Roeder et al., 1979; Engi and Evans, 1980; Sack, 1980). Apparently, spinel $\mathrm{Ti}^{4+}$ is more mobile than the trivalent cations under these metamorphic conditions as well.

Within individual samples, alteration of spinel margins was erratic, occurring in some grains and not in others. Margins of spinel inclusions were often subjected to metamorphic alteration, as illustrated by spinels from Sample 147-895E-2R-1, 36-39 cm, (transects of grains shown in Fig. 6; these occur within $\mathrm{An}_{85-87}$ plagioclase) and by analyses 4-IOC and 5-IOR from Sample 147-895D$7 \mathrm{R}-2,71-75 \mathrm{~cm}$, a core-rim pair of a $80 \times 100 \mu \mathrm{m}$ inclusion in olivine. These spinels show evidence of loss in $\mathrm{Ti}$ and a lowering in $\mathrm{Mg} \#$. Scowan et al. (1991) found that spinel inclusions in olivine from the Kilauea Iki lava lake became richer in $\mathrm{TiO}_{2}$ as the lava lake crystallized, indicating diffusive movement of $\mathrm{Ti}$ through olivine to the spinel; an opposite movement of Ti appears to have occurred here. Fractures in the host olivines and plagioclase, some lined with secondary clays, may have provided pathways for metamorphic fluids.

\section{Chemistry of "Fresher" Spinel}

To more closely approximate original Site 895 spinel compositional relationships, analyses considered significantly altered during the lower temperature metamorphic overprints were removed from consideration. Analyses within individual grains were considered acceptable for retention only if two or more analyses in a transect had similar Mg\# values, a value judgement based on the fact that the cores of all grains analyzed on transects had little zoning in $\mathrm{Mg \#}$ (e.g., Fig. 6)any analyses with significantly lower $\mathrm{Mg \#}$ than in these transects were excluded. Comparison with other analyses showed that the cores of some spinel grains smaller than $100 \mu \mathrm{m}$ were relatively low in $\mathrm{Mg}$ \# for a given $\mathrm{TiO}_{2}$ content (as shown in Table 2 banalyses 4-IOC and 5-IOR from Sample 147-895D-7R-2, 71-75 cm, in comparison to other analyses from this sample); these could reflect extensive higher temperature, metamorphic olivine-spinel $\mathrm{Mg}-\mathrm{Fe}^{2+}$ exchange (Ozawa, 1983) prior to lower amphibolite metamorphism, and they were excluded as well. The upper part of Table 1 and Figure 7 show the "uncorrected" data set, whereas the lower part of Table 1 and Figure 8 show the smaller, "fresher" data set.

After removal from consideration of analyses strongly affected by lower temperature metamorphic exchange, the crystal chemical variations within the Site 895 spinels become clearer and more systematic, both within individual samples and for the entire sample group (compare Figs. 7 and 8). Within the harzburgite samples, spinel composition is typical of those from abyssal, depleted harzburgite (Dick, 1989; Dick and Fisher, 1984; Dick and Bullen, 1984), with high Cr\#,

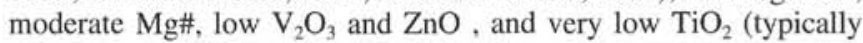
$\leq 0.05 \%$ ) and $\mathrm{Fe}^{3+} /\left(\mathrm{Cr}+\mathrm{Al}+\mathrm{Fe}^{3+}\right)\left(\mathrm{Fe}^{3+} \# ; \leq 0.05\right)$ (Tables 1, 2; Figs. 7,8 ). Internal zoning, other than the somewhat variable $\mathrm{V}_{2} \mathrm{O}_{3}$ and $\mathrm{ZnO}$, is limited within individual crystals, but variations exist grainto-grain within a single sample as well as between samples (Table 2; 

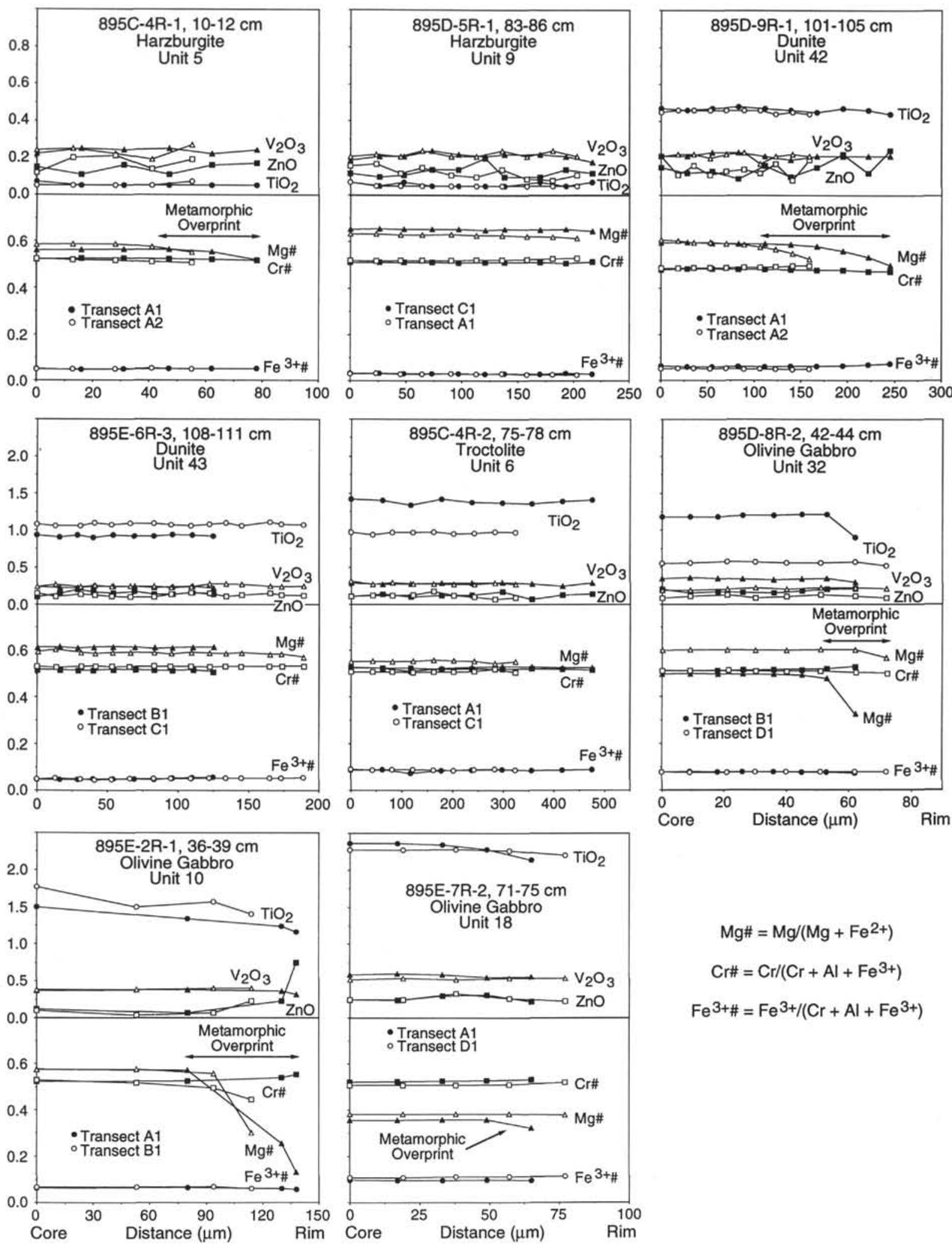

$$
\begin{gathered}
\mathrm{Mg} \#=\mathrm{Mg} /\left(\mathrm{Mg}+\mathrm{Fe}^{2+}\right) \\
\mathrm{Cr} \#=\mathrm{Cr} /\left(\mathrm{Cr}+\mathrm{Al}+\mathrm{Fe}^{3+}\right) \\
\mathrm{Fe}^{3+\#}=\mathrm{Fe}^{3+/\left(\mathrm{Cr}+\mathrm{Al}+\mathrm{Fe}^{3+}\right)}
\end{gathered}
$$

Figure 6. Core-to-rim electron microprobe transects across spinel grains from Site 895 samples, avoiding areas of obvious ferritchromite formation. Transect pairs are analyzed from grains within individual thin sections; representative analyses from these transects are given in Table 2. Post-crystallization metamor-

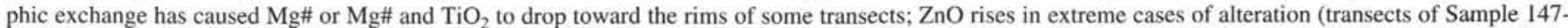
$895 \mathrm{E}-2 \mathrm{R}-1,36-39 \mathrm{~cm}$ ). Note that spinel $\mathrm{TiO}_{2}$ in the dunitic samples varies strongly from sample to sample, and that troctolites and olivine gabbros may show strong variance in $\mathrm{TiO}_{2}$ and $\mathrm{Mg} \#$ in spinels within a single thin section (Samples $147-895 \mathrm{C}-4 \mathrm{R}-2,75-78 \mathrm{~cm}$, and $147-895 \mathrm{D}-8 \mathrm{R}-2,42-44 \mathrm{~cm}$ ). 

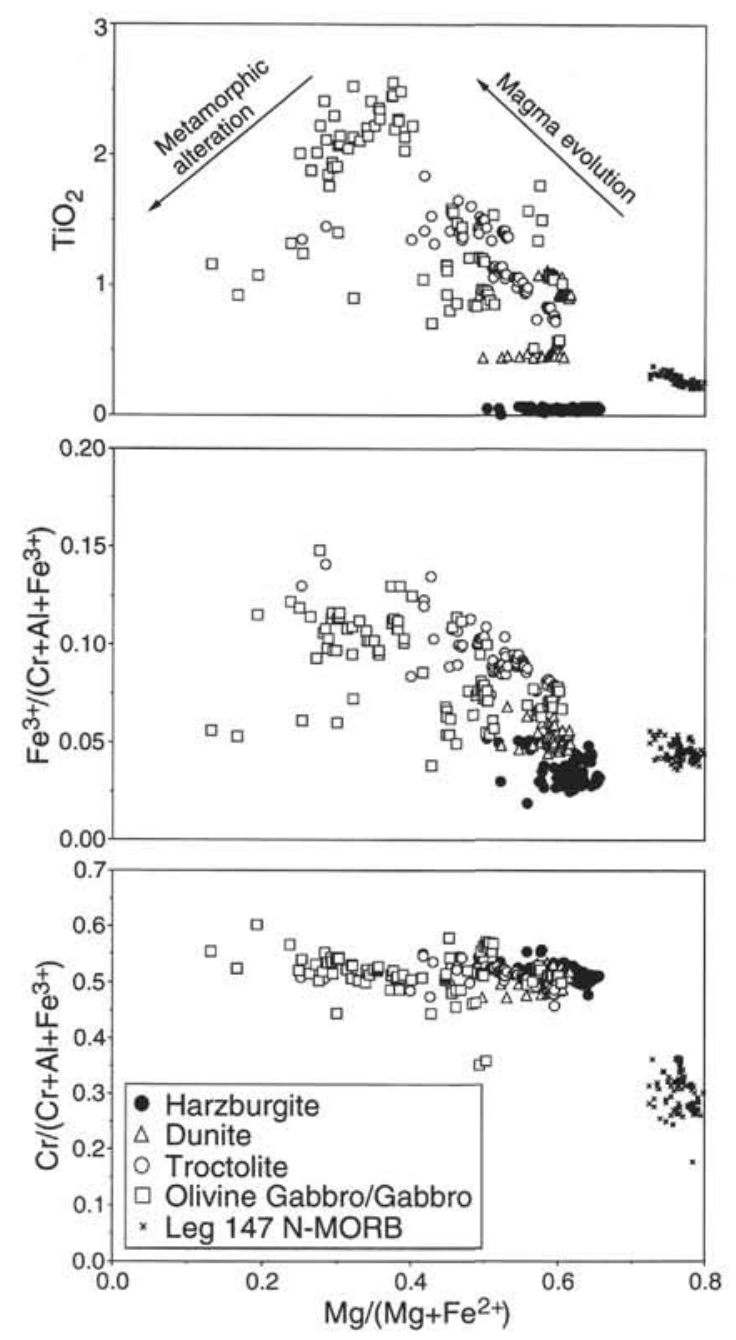

Figure 7. Electron microprobe analyses of spinels from the various Site 895 rock types. Also shown for comparison are $\mathrm{Cr}$-spinels analyzed from basaltic lava and dike samples from Site 894 that have an N-MORB compositional signal (Allan et al., this volume). Also shown are the inferred trends in $\mathrm{TiO}_{2}$ and $\mathrm{Mg} /\left(\mathrm{Mg}+\mathrm{Fe}^{2+}\right)$ for the troctolite and gabbro spinels from processes associated with magma evolution and post-crystallization metamorphic exchange (see text).

Fig. 6). In addition, analyses of vermicular spinel (analysis 5, Sample 147-895D-7R-2, 123-126 cm, and analysis 5, Sample 147-895D-4R$1,34-37 \mathrm{~cm}$, in Table 2) are little different than for other, larger subangular or subrounded spinels, other than having slightly higher $\mathrm{ZnO}$.

Dunitic spinels show substantial differences in composition compared with those in harzburgite. Although morphologically similar and overlapping in $\mathrm{Cr} \#$, they contain 10 to 20 times the amount of $\mathrm{TiO}_{2}$, with slightly higher average $\mathrm{Fe}^{3+} \#$ and $\mathrm{NiO}$ as well (Tables 1 , 2 ; Figs. 6, 8). Considering the two dunite samples as a group, there is a positive correlation between $\mathrm{TiO}_{2}$ and $\mathrm{Cr} \#$ (also found in other Site 895 dunites by Dick and Natland, this volume; see their fig. 10). A slight negative correlation between $\mathrm{TiO}_{2}$ and $\mathrm{Mg} \#$ is visible within dunite Sample 147-895E-6R-3, 108-111 cm. Like the spinels in harzburgite, the dunite spinels show only minor internal zoning and exhibit minor grain-to-grain variation within a single sample. Nevertheless, the two dunite samples analyzed have substantial differences in spinel $\mathrm{TiO}_{2}$ content, with the spinels in Sample 147-895E-6R-3, $108-111 \mathrm{~cm}$, having roughly twice the amount of $\mathrm{TiO}_{2}$ of those from
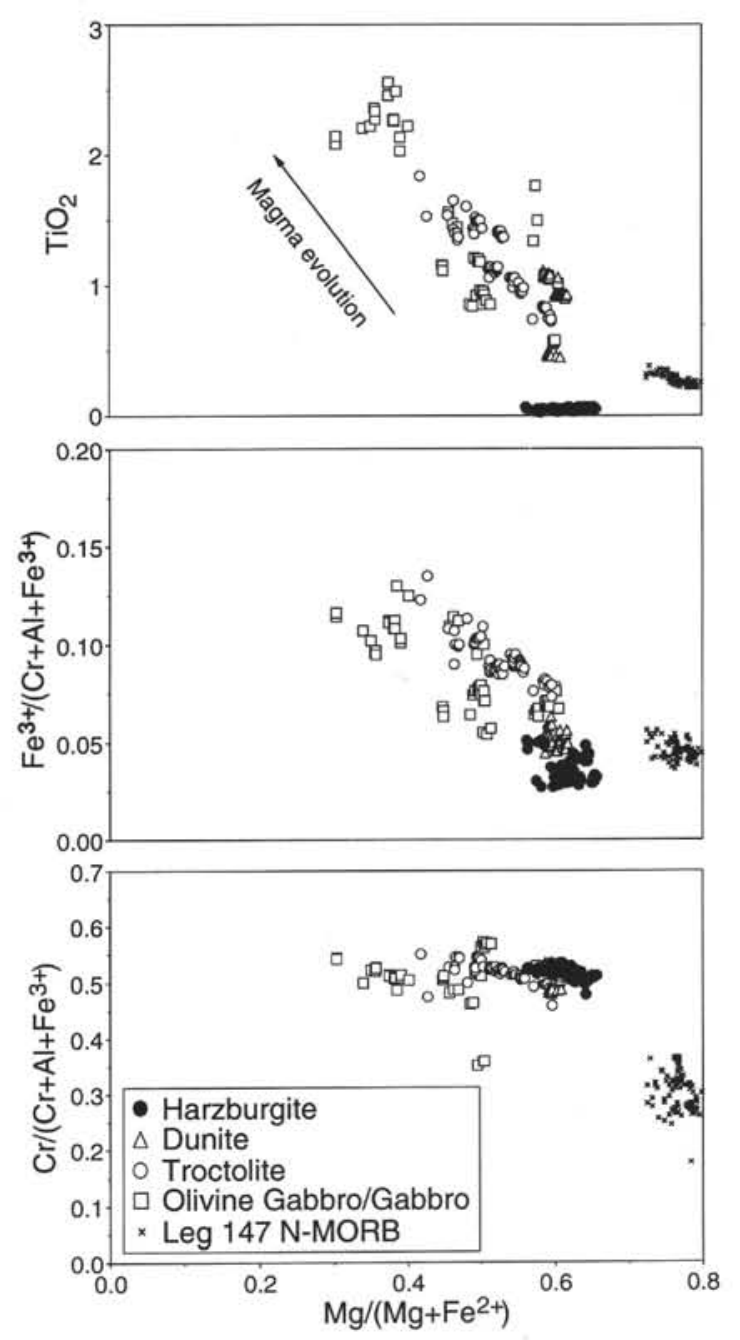

Figure 8. Electron microprobe analyses of "fresh" Cr-spinel cores from the various Site 895 rock types, omitting spinels affected by elemental exchange associated with metamorphic alteration and thereby giving a clearer view of the original Cr-spinel crystal chemistry.

Sample 147-895D-9R-1, 101-105 cm. Additional data reported by Dick and Natland (this volume) extend the range of Site 895 dunitic spinel $\mathrm{TiO}_{2}$ from $0.5 \%$ to $2.0 \%$. Of interest is that Sample 147-895E6R-3, 108-11 cm, (with Ti-rich spinel) occurs adjacent to a unit of troctolite, whereas Sample 147-895D-9R-1, 101-105 cm, (with relatively Ti-poor spinel) occurs between units of harzburgite and is not adjacent to gabbro or troctolite.

Within the gabbroic and troctolitic spinels, no systematic difference was noted in individual samples in the chemistry of spinels that occur as intergranular grains compared to those that occur as inclusions within olivine or plagioclase (Table 2). Systematic variations for the group are well illustrated in Figure 8, showing strong negative correlations of $\mathrm{TiO}_{2}, \mathrm{Fe}^{3+} \#$, and $\mathrm{Cr} \#$ with $\mathrm{Mg} \#$, with the latter correlation illustrating the crystal chemical effects of coupled $\mathrm{Fe}^{2+}-\mathrm{Cr}^{3+}$ and $\mathrm{Mg}^{2+}-\mathrm{Al}^{3+}$ substitution within spinel discussed previously. The negative correlation between $\mathrm{Cr} /(\mathrm{Cr}+\mathrm{Al})$ with $\mathrm{Mg} \#$, shown by Dick and Natland (this volume), is especially strong. Gabbro, olivine gabbro, and troctolitic spinels are strikingly enriched in $\mathrm{TiO}_{2}$ and broadly enriched in $\mathrm{V}_{2} \mathrm{O}_{3}$ over harzburgitic spinel, but show little overall difference in $\mathrm{NiO}$ and $\mathrm{ZnO}$ contents (Table 1; Fig. 8). Overall, the range in $\mathrm{Mg} \#$ exhibited by the gabbro and olivine gabbro spinels is substan- 
tial, overlapping in $\mathrm{Mg \#}$ with the dunitic and troctolitic spinels, but with many having a Mg\# of $<0.4$ (Fig. 8). The presence of spinel with low $\mathrm{Mg}$ \# is consistent with the relatively low Fo content of the associated olivine in both troctolitic and gabbroic samples, particularly in Sample 147-895D-7R-2, 71-75 cm (Table 1).

Sample 147-895D-7R-2, 71-75 cm, contains a broad range of spinel compositions, with the large number of grains analyzed (16) allowing a careful examination of spinel compositional systematics within a single thin-section sample of olivine gabbro. In considering the relatively unaltered cores, strong positive correlations of $\mathrm{TiO}_{2}$ exist with $\mathrm{V}_{2} \mathrm{O}_{3}$ and $\mathrm{MnO}$, with weaker positive correlations existing with $\mathrm{TiO}_{2}$ and $\mathrm{ZnO}$ and $\mathrm{Cr} \#$. In contrast, there is a strong negative correlation between spinel $\mathrm{Mg} \#$ and $\mathrm{TiO}_{2}$, and no correlations occur between $\mathrm{TiO}_{2}$ and $\mathrm{Fe}^{3+} \#$ and $\mathrm{NiO}$. These relationships are illustrated in Table 2 by analyses A1-C, 11-C, 16-C, D1-C, 30-C, and 33-C. Spinels from other gabbro samples share these relationships, with Sample 147-895D-8R-2, 42-44 cm, also showing a broad range in spinel composition (Table 1; analyses A1-C, B1-C, and D1-C in Table 2).

The Hole $895 \mathrm{C}$ troctolite samples most likely originate from the same intrusive event owing to their proximity and nearly identical physical character. They show poorer but similar correlations within their $\mathrm{Cr}$-spinel systematics when considered as a group. Tables 1 and 2 and Figure 6 show results of nine transects and eight other grains analyzed from Samples 147-895C-4R-2, 75-78 cm, 147-895C-4R-2, $126-128 \mathrm{~cm}$, and $147-895 \mathrm{C}-4 \mathrm{R}-1,63-68 \mathrm{~cm}$, with all showing the same curious lack of zoning within the large grains, some of which re skeletal. Plate $1 \mathrm{~F}$ shows a photomicrograph of the crystal of electron microprobe transect C1 from Sample 147-4R-2, 75-78 cm, (Fig. 6) which contains inclusions of irregular plagioclase $\left(\mathrm{An}_{84-86}\right)$ and clinopyroxene $\left(\mathrm{En}_{48-49}, \mathrm{Fs}_{0.04-0.05}, \mathrm{Wo}_{0.46-0.47}\right)$. Table 4 gives representative analyses of these grains, for which the unexsolved nature, small compositional range, and apparent protection from lower amphibolite to zeolite metamorphic alteration indicate that their compositions may approximate magmatic values.

A striking feature of both the troctolites and the gabbro samples is that fresh cores of individual spinel grains show little or no zoning, yet the differences in $\mathrm{TiO}_{2}$ between individual grains within a thin section can be quite significant. For example, the two electron microprobe transects shown for the troctolite Sample 147-895C-4R-2, 75$78 \mathrm{~cm}$, in Figure 6 differ by nearly $0.5 \%$ in $\mathrm{TiO}_{2}$, yet these two $0.5-$ $\mathrm{cm}$-sized grains are only about a centimeter apart in thin section. A similar distance separates the two spinel transects shown in Figure 6 from Sample 147-895D-8R-2, 42-44 cm. These grains show more than a two-fold variation in $\mathrm{TiO}_{2}$ despite sharing similar $\mathrm{Mg} \#$.

\section{DISCUSSION}

\section{Gabbroic Spinel Ti and $\mathrm{Fe}^{3+}$ Enrichment}

The spinels in gabbro and troctolite exhibit a large range in composition, with the dominant form of variation shown by the increase in $\mathrm{Ti}$ and $\mathrm{Fe}^{3+} \#$ with declining $\mathrm{Mg} \#$ (Table 1; Fig. 8). At low $\mathrm{Mg} \#$, $\mathrm{TiO}_{2}$ contents commonly exceed $2 \%$, similar to that found in other $\mathrm{Cr}$-spinels from abyssal and ophiolitic plagioclase-rich troctolites and plagioclase dunites (Cannat et al., 1990; Hekinian et al., 1993; Girardeau and Francheteau, 1993; Dick, 1989; Hebert et al., 1983). These $\mathrm{TiO}_{2}$ (and $\mathrm{Fe}^{3+\#)}$ values are much higher than those observed in N-MORB Cr-spinel (Fig. 8; Allan et al., this volume; Allan et al., 1988) and are similar in enrichment to those found in oceanic alkali basalt (Allan et al., 1993, 1994; Batiza and Vanko, 1984). Nevertheless, numerous pieces of evidence require that the parental basaltic melts to the gabbroic $\mathrm{Cr}$-spinels be of an N-MORB compositional character. This evidence includes (1) bulk composition of Site 895 gabbroic rocks, which are depleted in incompatible elements (Gillis, Mével, Allan, et al., 1993; J. Allan, unpub. data); (2) electron and ion microprobe analysis of gabbroic clinopyroxene (Dick and Natland, this volume; H.J.B. Dick and J. Natland, unpub. data), which shows compositions depleted in $\mathrm{Ti}$ and the rare earth elements; (3) low $\mathrm{Ti}$ contents of the clinopyroxene inclusions in skeletal, troctolite spinel, with compositions typical or lower than that of N-MORB clinopyroxene (e.g., Allan et al., 1987; Perfit and Fornari, 1983; Mazzullo and Bence, 1976); and (4) K-poor, An-rich contents of the plagioclases analyzed, especially considering the inclusions in troctolitic spinel.

Given that the impregnating melt is of N-MORB character, the strong negative correlation of $\mathrm{Ti}$ and $\mathrm{Fe}^{3+} \#$ with $\mathrm{Mg} \#$ most likely reflects spinel crystallizing from or reequilibrating with melt that is evolving as a result of crystallization and melt-wall rock interaction. Both processes will increase melt $\mathrm{Ti}$ and $\mathrm{Fe}^{3+}$ as long as the magma mass is decreasing, because these elements are excluded from plagioclase and olivine. The large variations in spinel $\mathrm{TiO}_{2}$ within a single thin section argue that these variations are related to spinel reequilibrating with increasingly small pockets of intercrystalline, evolving melt during cooling after or during melt impregnation. The presence of evolved melt is supported by the relatively fayalitic nature of the adjacent olivines in the gabbros, particularly within Sample 147895D-7R-2, 71-75 cm (Tables 1 and 3). Within other samples, substantial variation in spinel $\mathrm{Ti}$ with little variation in spinel Mg\# (such as shown by transects B1 and D1 of Sample 147-895D-8R-2, 42-44 $\mathrm{cm}$, in Fig. 6) may reflect varying degrees of wall-rock interaction, with the $\mathrm{Mg}$ \# buffered by the surrounding peridotite as the volume of melt decreased during crystallization.

\section{Implications of Spinel in Dunite}

The composition of the spinels in dunite exerts strong constraints on models of dunite origin. The high $\mathrm{TiO}_{2}$ of the dunite spinel, approximately 10 times that of Hess Deep harzburgite spinel, rules out the possibility that the dunites analyzed here represent residual peridotite that has been melted to a pyroxene-out condition, as $\mathrm{Ti}$ is incompatible during mantle partial melting (e.g., Dick, 1989). In addition, the $\mathrm{Cr} \#$ of dunite spinel is similar to that of harzburgite spinel (Table 1; Fig. 8), whereas it should be higher if the dunites represent residua from greater partial melting (Dick and Bullen, 1984). The lack of cumulate mineral textures, the similarly high $\mathrm{Mg} \#$ of the dunite and harzburgite tectonite olivines, and the typical stratigraphic occurrence of dunite both above and below gabbro are incompatible with the Hess Deep dunites originating as simple cumulates from a magma chamber, as inferred for similar loose dunite and troctolite dredged from the Garrett Fracture Zone (Hebert et al., 1983). Instead, the high Ti contents of the dunite spinels, and the proximity of dunite to melt impregnations, indicate that the dunites formed from meltwall rock interaction, with a net outflow of $\mathrm{Ti}$ into the wall rock from the melt (Cannat et al., 1990). The high Ti contents of the dunite spinels are similar to those found within some ophiolitic podiform chromite bodies; these bodies are enclosed within dunitic envelopes that are themselves surrounded by harzburgite with Ti-poor spinel and are proposed to result from melt-wall rock interaction surrounding channels of upward melt flow (Dick, 1977, 1989; Arai and Yurimoto, in press; Leblanc and Ceuleneer, 1992; Lago et al., 1982).

The impregnated Hess Deep peridotites clearly illustrate processes of melt-wall rock disequilibrium, indicating that the melts could not have been generated from a similar peridotite in the immediate vicinity. The easiest explanation for the origin of this disequilibrium is to propose that the invading melt originated at a pressure substantially greater than that during melt impregnation. Melt originating from a given peridotite composition will have lower $a_{\mathrm{SiO}_{2}}$ at greater pressures of partial melting (e.g., Jacques and Green, 1980; Basaltic Volcanism Study Project, 1981); therefore, melt generated at higher pressure that is percolating through similar peridotite at substantially lower pressure will necessarily be out of equilibrium with the surrounding wall rock. The result during melt-wall rock interaction will thermodynamically drive dissolution of relatively $\mathrm{Si}$-rich phases, such as orthopyroxene and clinopyroxene, and drive precipitation of relatively Si-poor phases, such as olivine and spinel (Kelemen, 1990; Nicolas, 1989; Fisk, 1986; Dick, 1977; Sinton, 1977), in order to in- 
Table 3. Representative olivine analyses.

\begin{tabular}{|c|c|c|c|c|c|c|c|c|c|c|c|c|c|}
\hline $\begin{array}{l}\text { Rock: } \\
\text { Hole: } \\
\text { Core, section: } \\
\text { Interval }(\mathrm{cm}) \text { : } \\
\text { Analysis: }\end{array}$ & $\begin{array}{c}\text { OG } \\
895 \mathrm{E} \\
2 \mathrm{R}-1 \\
36-39 \\
2\end{array}$ & $\begin{array}{c}\text { OG } \\
895 \mathrm{E} \\
2 \mathrm{R}-1 \\
36-39 \\
4\end{array}$ & $\begin{array}{c}\text { OG } \\
895 \mathrm{D} \\
7 \mathrm{R}-2 \\
71-75 \\
2\end{array}$ & $\begin{array}{c}\text { OG } \\
895 \mathrm{D} \\
7 \mathrm{R}-2 \\
71-75 \\
3\end{array}$ & $\begin{array}{c}\mathrm{T} \\
895 \mathrm{C} \\
4 \mathrm{R}-1 \\
63-68 \\
1\end{array}$ & $\begin{array}{c}\mathrm{T} \\
895 \mathrm{C} \\
4 \mathrm{R}-1 \\
63-68 \\
3\end{array}$ & $\begin{array}{c}T \\
895 C \\
4 R-1 \\
63-68 \\
7\end{array}$ & $\begin{array}{c}\mathrm{T} \\
895 \mathrm{C} \\
4 \mathrm{R}-2 \\
75-58 \\
1\end{array}$ & $\begin{array}{c}\mathrm{T} \\
895 \mathrm{C} \\
4 \mathrm{R}-2 \\
75-58 \\
4\end{array}$ & $\begin{array}{c}\mathrm{T} \\
895 \mathrm{C} \\
4 \mathrm{R}-2 \\
75-58 \\
5\end{array}$ & $\begin{array}{c}\mathrm{T} \\
895 \mathrm{C} \\
4 \mathrm{R}-2 \\
75-58 \\
6\end{array}$ & $\begin{array}{c}\mathrm{T} \\
895 \mathrm{C} \\
4 \mathrm{R}-2 \\
75-58 \\
7\end{array}$ & $\begin{array}{c}\mathrm{T} \\
895 \mathrm{C} \\
4 \mathrm{R}-2 \\
126-128 \\
8\end{array}$ \\
\hline $\begin{array}{l}\mathrm{SiO}_{2} \\
\mathrm{FeO} \\
\mathrm{MnO} \\
\mathrm{NiO} \\
\mathrm{MgO} \\
\mathrm{CaO} \\
\text { Total }\end{array}$ & $\begin{array}{r}40.29 \\
11.59 \\
0.18 \\
0.26 \\
47.79 \\
0.00 \\
100.11\end{array}$ & $\begin{array}{r}40.17 \\
11.43 \\
0.13 \\
0.26 \\
47.97 \\
0.03 \\
100.00\end{array}$ & $\begin{array}{r}39.29 \\
15.08 \\
0.21 \\
0.22 \\
45.00 \\
0.03 \\
99.83\end{array}$ & $\begin{array}{r}39.40 \\
16.03 \\
0.28 \\
0.22 \\
44.34 \\
0.03 \\
100.29\end{array}$ & $\begin{array}{r}40.32 \\
10.39 \\
0.18 \\
0.30 \\
49.00 \\
0.04 \\
100.23\end{array}$ & $\begin{array}{r}40.32 \\
10.79 \\
0.17 \\
0.27 \\
48.49 \\
0.05 \\
100.08\end{array}$ & $\begin{array}{r}40.48 \\
10.89 \\
0.15 \\
0.30 \\
48.26 \\
0.06 \\
100.13\end{array}$ & $\begin{array}{r}40.25 \\
9.86 \\
0.14 \\
0.31 \\
48.99 \\
0.03 \\
99.58\end{array}$ & $\begin{array}{r}40.04 \\
10.84 \\
0.20 \\
0.29 \\
48.11 \\
0.05 \\
99.52\end{array}$ & $\begin{array}{r}40.38 \\
10.16 \\
0.15 \\
0.29 \\
48.38 \\
0.07 \\
99.42\end{array}$ & $\begin{array}{r}40.44 \\
10.43 \\
0.18 \\
0.29 \\
48.77 \\
0.03 \\
100.13\end{array}$ & $\begin{array}{r}40.44 \\
10.56 \\
0.18 \\
0.32 \\
48.33 \\
0.05 \\
99.88\end{array}$ & $\begin{array}{r}40.27 \\
10.41 \\
0.16 \\
0.33 \\
48.36 \\
0.05 \\
99.58\end{array}$ \\
\hline $\begin{array}{l}\text { Fo } \\
\text { Near Cr-spinel }\end{array}$ & $\begin{array}{l}0.880 \\
12\end{array}$ & $\begin{array}{l}0.882 \\
0\end{array}$ & $\begin{array}{l}0.842 \\
4\end{array}$ & $\begin{array}{l}0.831 \\
2,3\end{array}$ & 0.894 & $\begin{array}{l}0.889 \\
5\end{array}$ & $\begin{array}{l}0.888 \\
8\end{array}$ & $\begin{array}{l}0.899 \\
\text { Al }\end{array}$ & $\begin{array}{l}0.888 \\
\text { B2 }\end{array}$ & $\begin{array}{l}0.895 \\
\mathrm{~B} 1\end{array}$ & $\begin{array}{l}0.893 \\
\mathrm{Cl}\end{array}$ & $\begin{array}{l}0.891 \\
\mathrm{C} 2\end{array}$ & $\begin{array}{l}0.892 \\
\text { AAl }\end{array}$ \\
\hline
\end{tabular}

\begin{tabular}{|c|c|c|c|c|c|c|c|c|c|c|c|c|c|c|}
\hline $\begin{array}{l}\text { Rock: } \\
\text { Hole: } \\
\text { Core, section: } \\
\text { Interval }(\mathrm{cm}) \text { : } \\
\text { Analysis: }\end{array}$ & $\begin{array}{c}\mathrm{T} \\
895 \mathrm{C} \\
4 \mathrm{R}-2 \\
126-128 \\
10\end{array}$ & $\begin{array}{c}\mathrm{T} \\
895 \mathrm{C} \\
4 \mathrm{R}-2 \\
126-128 \\
11\end{array}$ & $\begin{array}{c}D \\
895 E \\
6 R-3 \\
108-111 \\
3\end{array}$ & $\begin{array}{c}\mathrm{D} \\
895 \mathrm{E} \\
6 \mathrm{R}-3 \\
108-111 \\
4\end{array}$ & $\begin{array}{c}\mathrm{HZ} \\
895 \mathrm{~A} \\
2 \mathrm{R}-1 \\
7-10 \\
2\end{array}$ & $\begin{array}{c}\mathrm{HZ} \\
895 \mathrm{~A} \\
2 \mathrm{R}-1 \\
7-10 \\
7\end{array}$ & $\begin{array}{c}\mathrm{HZ} \\
895 \mathrm{C} \\
4 \mathrm{R}-1 \\
10-12 \\
2\end{array}$ & $\begin{array}{c}\mathrm{HZ} \\
895 \mathrm{D} \\
2 \mathrm{R}-1 \\
123-126 \\
1\end{array}$ & $\begin{array}{c}\mathrm{HZ} \\
895 \mathrm{D} \\
4 \mathrm{R}-1 \\
34-37 \\
12\end{array}$ & $\begin{array}{c}\mathrm{HZ} \\
895 \mathrm{D} \\
5 \mathrm{R}-1 \\
83-86 \\
3\end{array}$ & $\begin{array}{c}\mathrm{HZ} \\
895 \mathrm{D} \\
5 \mathrm{R}-1 \\
83-86 \\
7\end{array}$ & $\begin{array}{c}\mathrm{HZ} \\
895 \mathrm{D} \\
7 \mathrm{R}-2 \\
123-126 \\
6\end{array}$ & $\begin{array}{c}\mathrm{HZ} \\
895 \mathrm{D} \\
7 \mathrm{R}-2 \\
123-126 \\
9\end{array}$ & $\begin{array}{c}\mathrm{HZ} \\
895 \mathrm{D} \\
7 \mathrm{R}-2 \\
123-126 \\
11\end{array}$ \\
\hline $\begin{array}{l}\mathrm{SiO}_{2} \\
\mathrm{FeO} \\
\mathrm{MnO} \\
\mathrm{NiO} \\
\mathrm{MgO} \\
\mathrm{CaO} \\
\text { Total }\end{array}$ & $\begin{array}{r}40.62 \\
10.39 \\
0.13 \\
0.35 \\
48.51 \\
0.07 \\
100.06\end{array}$ & $\begin{array}{r}40.54 \\
10.15 \\
0.16 \\
0.32 \\
48.63 \\
0.05 \\
99.84\end{array}$ & $\begin{array}{r}40.73 \\
9.88 \\
0.16 \\
0.38 \\
50.46 \\
0.15 \\
101.76\end{array}$ & $\begin{array}{r}40.87 \\
9.47 \\
0.14 \\
0.40 \\
50.54 \\
0.18 \\
101.60\end{array}$ & $\begin{array}{r}40.21 \\
8.88 \\
0.10 \\
0.40 \\
50.21 \\
0.02 \\
99.82\end{array}$ & $\begin{array}{r}40.76 \\
8.51 \\
0.13 \\
0.43 \\
50.30 \\
0.06 \\
100.18\end{array}$ & $\begin{array}{r}40.54 \\
8.86 \\
0.10 \\
0.41 \\
49.94 \\
0.03 \\
99.89\end{array}$ & $\begin{array}{r}40.15 \\
9.01 \\
0.12 \\
0.44 \\
49.60 \\
0.06 \\
99.38\end{array}$ & $\begin{array}{r}40.38 \\
8.91 \\
0.11 \\
0.35 \\
49.75 \\
0.05 \\
99.54\end{array}$ & $\begin{array}{r}41.05 \\
8.17 \\
0.12 \\
0.39 \\
50.45 \\
0.08 \\
100.25\end{array}$ & $\begin{array}{r}41.06 \\
8.63 \\
0.15 \\
0.36 \\
50.00 \\
0.03 \\
100.22\end{array}$ & $\begin{array}{r}40.18 \\
8.88 \\
0.11 \\
0.44 \\
49.87 \\
0.04 \\
99.53\end{array}$ & $\begin{array}{r}40.71 \\
8.94 \\
0.16 \\
0.40 \\
50.29 \\
0.06 \\
100.55\end{array}$ & $\begin{array}{r}40.39 \\
9.09 \\
0.14 \\
0.41 \\
50.02 \\
0.01 \\
100.05\end{array}$ \\
\hline $\begin{array}{l}\text { Fo } \\
\text { Near Cr-spinel }\end{array}$ & $\begin{array}{l}0.893 \\
\mathrm{BB} 2\end{array}$ & $\begin{array}{l}0.895 \\
\mathrm{BB} 1\end{array}$ & $\begin{array}{l}0.901 \\
\mathrm{~B} 1\end{array}$ & $\begin{array}{l}0.905 \\
\mathrm{Cl}\end{array}$ & $\begin{array}{l}0.910 \\
\text { A1 }\end{array}$ & $\begin{array}{l}0.913 \\
\mathrm{CI}\end{array}$ & $\begin{array}{l}0.909 \\
\text { A2 }\end{array}$ & $\begin{array}{l}0.907 \\
\mathrm{~A} 1\end{array}$ & $\begin{array}{l}0.909 \\
5\end{array}$ & $\begin{array}{l}0.917 \\
\text { B }\end{array}$ & ${ }_{\mathrm{C}}^{0.912}$ & $\begin{array}{l}0.909 \\
\text { B1 }\end{array}$ & $\begin{array}{l}0.909 \\
\mathrm{Cl}\end{array}$ & $\begin{array}{l}0.907 \\
5\end{array}$ \\
\hline
\end{tabular}

Notes: Electron microprobe analyst, J. Allan. $\mathrm{OG}=$ olivine gabbro; $\mathrm{T}=$ troctolite; $\mathrm{D}=$ dunite; $\mathrm{HZ}=$ harzburgite; Fo $=$ olivine forsterite content. Labels of adjacent analyzed spinel grains are also given.

crease the $a_{\mathrm{SiO}_{2}}$ of the ascending melt. Recent geochemical modeling (Kelemen et al., in press) indicates that the wall rock may in fact be entirely replaced during this process, with the precipitation of large amounts of olivine. Therefore, dunitic pods containing spinel within mantle harzburgite may be relicts of melt-upflow channels, even where no obvious melt impregnations are present. Bodies of isolated dunite, sampled during Leg 147 without direct evidence of adjacent impregnated melt (such as the Hole 895D Unit 42 dunite of Sample 147-895D-9R-1, 101-105 cm), may have shared such an origin.

Other recent work regarding the origin of abundant $\mathrm{Cr}$-spinel in podiform chromite bodies suggests that magma mixing, involving Sirich secondary melt generated during melt-wall rock interaction, may be involved in the rapid precipitation of $\mathrm{Cr}$-spinel during basaltic melt-peridotite wall rock interaction (Arai and Yurimoto, in press). The skeletal troctolite spinels from Hole 895C, apparently rapidly grown, probably reflect crystallization by such a process; their widely varying $\mathrm{Ti}$ contents provide evidence for a complex series of melts that vary in composition because of fractionation and disequilibrium exchange on a centimeter-sized scale (Cannat et al., 1990; Dick and Kelemen, 1991; Leblanc and Ceuleneer, 1992; Kelemen et al., in press).

\section{Constraints on Conditions and Location of Impregnation}

The nature of occurrence, relative stratigraphy, and composition of the Site 895 spinel impose constraints on the physical conditions and possible location of melt impregnation. The conditions must be such to allow dispersal of exotic melt away from melt channels into intergranular networks in the surrounding peridotite, thereby forming dunite with troctolite, which sometimes was preserved on the margins of the melt channels. The relatively evolved nature and extensive recovery of some of the gabbroic rocks (more than $1 \mathrm{~m}$ of continuous core for Units 2 and 10, Hole 895E, and more than $0.5 \mathrm{~m}$ for Unit 28 , Hole 895E; Gillis, Mével, Allan, et al., 1993) indicate that much of the melt must have been channeled through dikelike bodies. The ambient temperature of the wall rock must have been low enough to preserve evidence of obvious disequilibria within the gabbroic and troctolitic rocks, as represented by individual samples with strongly variable spinel Ti contents. Therefore, during impregnation, the peridotite was (1) within the plagioclase stability field; (2) substantially shallower than typical depths associated with MORB genesis (e.g., significantly shallower than about 8-9 kbar pressure or about $25 \mathrm{~km}$ depth; Walter and Presnall, 1994; Presnall and Hoover, 1987; Kinzler and Grove, 1992a, 1992b; Nui and Batiza, 1991; McKenzie and Bickle, 1988; Klein and Langmuir, 1987) in order to account for melt-wall rock disequilibria; (3) no warmer than the liquidus of the impregnating melt (to preserve spinel compositional disequilibria); and (4) warm enough to allow melt impregnation by ductile deformation (Kennedy et al., this volume, found no evidence for microstructural olivine deformation in the dunites, requiring emplacement temperatures of at least $800^{\circ} \mathrm{C}$ ). In addition, melt impregnation occurred most probably near the base of the crust, in order to allow uplift and exposure on the seafloor at a point that is relatively close to the spreading ridge.

Olivine-spinel geothermometry (Sack and Ghiorso, 1991a, 1991b) provides minimum melt impregnation temperatures (Table 1). These temperatures, derived from adjacent olivine-spinel pairs, represent metamorphic cooling reequilibration temperatures. To minimize compositional variation caused by post-crystallization exchange, the cores of adjacent spinel-olivine pairs were used. Sample 147-895E-2R-1, 36-39 cm, an olivine gabbro, gives the lowest temperatures, which most likely reflect spinel reequilibration during hydrothermal alteration that produced the $\mathrm{Zn}$-rich spinel margins. Otherwise, maximum temperatures of about $880^{\circ}-900^{\circ} \mathrm{C}$ were found for the troctolites and dunites, with somewhat lower maximum temperatures given by the olivine gabbros and harzburgites. These temperatures represent minimum estimates for ambient wall-rock temperature during impregnation, and almost certainly reflect some 
Table 4. Selected plagioclase and clinopyroxene analyses.

\begin{tabular}{|c|c|c|c|c|c|c|c|}
\hline Rock: & OG & OG & OG & OG & OG & $\mathrm{T}$ & $\mathrm{T}$ \\
\hline Hole: & 895D & $895 \mathrm{D}$ & 895D & $895 \mathrm{E}$ & $895 \mathrm{E}$ & $895 \mathrm{C}$ & $895 \mathrm{C}$ \\
\hline Core, section: & $7 \mathrm{R}-2$ & $7 R-2$ & $7 \mathrm{R}-2$ & $2 \mathrm{R}-1$ & $2 \mathrm{R}-1$ & $4 \mathrm{R}-2$ & $4 \mathrm{R}-2$ \\
\hline Interval $(\mathrm{cm})$ : & $71-75$ & $71-75$ & $71-75$ & $36-39$ & $36-39$ & $75-78$ & $75-78$ \\
\hline Analysis \#: & 2 & 3 & 4 & 1 & 3 & 2I & $4 \mathrm{I}$ \\
\hline \multicolumn{8}{|l|}{ Plagioclase } \\
\hline $\mathrm{SiO}_{2}$ & 48.13 & 46.30 & 46.97 & 46.62 & 46.53 & 47.00 & 46.47 \\
\hline $\mathrm{Al}_{2} \mathrm{O}_{3}$ & 33.45 & 34.69 & 34.35 & 34.48 & 34.73 & 34.49 & 34.82 \\
\hline $\mathrm{FeO}$ & 0.17 & 0.15 & 0.17 & 0.27 & 0.29 & 0.19 & 0.16 \\
\hline $\mathrm{MgO}$ & 0.02 & 0.02 & 0.02 & 0.16 & 0.09 & 0.03 & 0.03 \\
\hline $\mathrm{CaO}$ & 15.93 & 17.64 & 16.78 & 17.69 & 17.58 & 17.00 & 17.52 \\
\hline $\mathrm{Na}_{2} \mathrm{O}$ & 2.49 & 1.61 & 1.91 & 1.48 & 1.65 & 1.74 & 1.59 \\
\hline $\mathrm{K}_{2} \mathrm{O}$ & 0.00 & 0.02 & 0.01 & 0.00 & 0.02 & 0.01 & 0.01 \\
\hline Total & 100.19 & 100.42 & 100.20 & 100.70 & 100.87 & 100.46 & 100.59 \\
\hline An & 77.9 & 85.7 & 82.9 & 86.9 & 85.4 & 84.3 & 85.9 \\
\hline
\end{tabular}

\begin{tabular}{lccc}
\hline Rock: & $\mathrm{T}$ & $\mathrm{T}$ & $\mathrm{T}$ \\
Hole: & $895 \mathrm{C}$ & $895 \mathrm{C}$ & $895 \mathrm{C}$ \\
Section: & $4 \mathrm{R} 2$ & $4 \mathrm{R} 2$ & $4 \mathrm{R} 2$ \\
Interval $(\mathrm{cm}):$ & $75-78$ & $75-78$ & $75-78$ \\
Analysis \#: & $5 \mathrm{I}$ & $6 \mathrm{I}$ & $7 \mathrm{I}$ \\
\hline & & & \\
\hline Clinopyroxene & & & \\
$\mathrm{SiO}_{2}$ & 51.27 & 52.09 & 51.42 \\
$\mathrm{TiO}_{2}$ & 0.72 & 0.69 & 0.69 \\
$\mathrm{Al}_{2} \mathrm{O}_{3}$ & 3.10 & 2.60 & 2.97 \\
$\mathrm{Cr}_{2} \mathrm{O}_{3}$ & 1.23 & 0.92 & 0.93 \\
$\mathrm{FeO}$ & 3.29 & 2.66 & 3.02 \\
$\mathrm{MnO}$ & 0.07 & 0.08 & 0.09 \\
$\mathrm{NiO}$ & 0.08 & 0.02 & 0.06 \\
$\mathrm{MgO}$ & 16.98 & 17.15 & 16.75 \\
$\mathrm{CaO}$ & 22.45 & 23.28 & 22.99 \\
$\mathrm{Na}{ }_{2} \mathrm{O}$ & 0.37 & 0.35 & 0.35 \\
$\mathrm{~K}_{2} \mathrm{O}$ & 0.00 & 0.00 & 0.01 \\
$\mathrm{Total}$ & 99.56 & 99.84 & 99.28 \\
$\mathrm{Mg} \#$ & 0.902 & 0.920 & 0.908 \\
$\mathrm{En}$ & 0.486 & 0.485 & 0.479 \\
$\mathrm{Fs}$ & 0.053 & 0.042 & 0.048 \\
$\mathrm{WO}$ & 0.462 & 0.473 & 0.473 \\
\hline
\end{tabular}

Notes: Electron microprobe analyst, J. Allan. $\mathrm{OG}=$ olivine gabbro, $\mathrm{T}=$ troctolite; $\mathrm{An}=$ anorthite content; $\mathrm{Mg} \#=\mathrm{Mg} /\left(\mathrm{Mg}+\mathrm{Fe}{ }^{2+}\right) ; \mathrm{En}=\mathrm{enstatite}$ content; Fs $=$ ferrosilite content; Wo = wollastonite content.

amounts of spinel-olivine $\mathrm{Mg}-\mathrm{Fe}^{2+}$ exchange during extended cooling.

The temperature of the wall rock during melt impregnation may also be estimated by approximating magmatic temperatures of MORB-like melts in equilibrium with the most $\mathrm{Fe}$-rich olivine compositions in the gabbroic melt impregnations (unzoned olivine grains of $\mathrm{Fo}_{83-84}$ composition in Sample 147-895D-7R-2, 71-75 cm) at appropriate pressures. These impregnating melts would have rapidly solidified if they had cooled below a point where they were in equilibrium with the olivine phase; indeed, the impregnating melts may be envisioned as existing within a crucible made up mostly of olivine with subordinate orthopyroxene. The experimental study by Bender et al. (1978), in which phase relations and compositions of a primitive FAMOUS basalt from 1 bar to $15 \mathrm{kbar}$ were examined, provides a basis for approximating the temperature of the melts in equilibrium with olivine of $\mathrm{Fo}_{83-84}$ composition in the last stages of cooling, and therefore an estimate of the wall-rock temperature during melt impregnation. The FAMOUS basalt used by Bender et al. (1978) is an appropriate choice for comparison, as both it and the Leg 147 Hess Deep gabbroic rocks are relatively depleted in incompatible elements and are interpreted to have been the products of large amounts of partial melting from depleted sources (Dick and Natland, this volume). Interpolating between the 1 bar and 6 kbar Bender et al. (1978) olivine compositional data and comparing those data with the olivine data presented here leads to wall-rock estimates of about $1230^{\circ} \mathrm{C}$ at 3 to $5 \mathrm{kbar}$ model pressures for the melt impregnation represented by Sample 147-895D-7R-2, 71-75 cm.

This temperature estimate may have been affected by post-crystallization $\mathrm{Mg}-\mathrm{Fe}^{2+}$ exchange between the olivine of the melt impreg- nation and the olivine of the surrounding dunitic envelop, thereby raising the $\mathrm{Mg} \#$ of the gabbroic olivine. As a result, this temperature estimate is most appropriately viewed as a maximum wall-rock temperature during emplacement. Owing to the small volume of spinel in this sample (shown in Pl. lG), the olivine composition was most likely little affected by post-crystallization, high-temperature metamorphic exchange with spinel.

Overall, the evidence suggests that melt impregnation occurred as a sequence of events, with injection occurring as dikes, irregular melt concentrations, and small-scale, intergranular diffusive flow. Melt injection probably took place at temperatures well above $900^{\circ} \mathrm{C}$ and most likely approaching $1230^{\circ} \mathrm{C}$, at depths below but near the base of the crust ( 4 to $10 \mathrm{~km}$ depth). These conditions are roughly similar to those proposed by Nicolas (1989) to explain the origin of similar melt-impregnated dunite bodies in ophiolitic harzburgite. Our results do not constrain whether melt impregnation took place under the ridge or slightly off-axis (both could have, in fact, taken place).

\section{CONCLUSIONS}

1. Depleted, residual harzburgites, now exposed by faulting at Hess Deep and sampled by coring during ODP Leg 147, were repeatedly impregnated with exotic N-MORB melt at depths of $<10 \mathrm{~km}$ and temperatures substantially above $900^{\circ} \mathrm{C}$ and probably approaching $1230^{\circ} \mathrm{C}$. Melt injection was accomplished by both diking and intergranular flow and occurred underneath or near the adjacent East Pacific Rise. This sequence of events is now represented by a generalized recovered stratigraphy of harzburgite-dunite-troctolitegabbro-troctolite-dunite-harzburgite. 
2. Ti contents in associated dunitic spinel are 10-20 times as high as in harzburgitic spinel, which indicates together with the mapped stratigraphy that the dunite recovered during Leg 147 is a relic of melt-wall rock interaction associated with melt impregnation. This process produced halos of dunite around residual bodies of gabbroic rock and possible residual dunite "trails" of melt migration through peridotite.

3. "Fresh" gabbroic spinel compositions vary widely $\left(\mathrm{TiO}_{2}\right.$, $0.55 \%-2.56 \% ; \mathrm{Mg} \#, 0.30-0.60$ ), commonly within individual samples. This wide range in composition shows that the impregnating melts became locally evolved, with Ti-enriched melts produced on a centimeter scale by both crystal fractionation and local melt-rock interaction.

4. $\mathrm{Ti}$ in spinel is the element most easily analyzed by the electron microprobe for monitoring mass exchange of incompatible elements during melt-wall rock interaction, and was mobile during both melt impregnation and during the subsequent greenschist-zeolite metamorphic overprint.

5. Sharp increases in $\mathrm{ZnO}$ (to $0.75 \%$ ) in the metamorphically altered rims of gabbro and troctolite spinels indicate that $\mathrm{Zn}$-rich hydrothermal fluids may have been present during the alteration of melt-impregnated peridotites, with the gabbroic melt impregnations providing possible preferential conduits for fluid flow.

\section{ACKNOWLEDGMENTS}

We thank the SEDCO/BP 471 crew, ODP engineers, and ODP technical staff for their Herculean efforts to recover these samples. J. Allan and H. Dick are grateful to NSF and USSAC for supporting these studies, to the Leg 147 co-chief scientists and other members of the shipboard party (especially S. Arai and J. Natland) for many intensive and illuminating discussions on peridotite spinels, and to R. Guillemette and S. Lakkapragada for analytical assistance. This manuscript was substantially improved from thorough reviews by $\mathrm{G}$. Cawthorne, P. Meyer, and an anonymous reviewer.

\section{REFERENCES}

Allan, J.F., 1992. Cr-Spinel as a petrogenetic indicator: deducing magma composition from spinels in highly altered basalts from the Japan Sea, Sites 794 and 797. In Tamaki, K., Suyehiro, K., Allan, J., McWilliams, M., et al., Proc. ODP, Sci. Results, 127/128 (Pt. 2): College Station, TX (Ocean Drilling Program), 837-847.

Allan, J.F., Batiza, R., and Lonsdale, P.F., 1987. Petrology and chemistry of lavas from seamounts flanking the East Pacific Rise axis, $21^{\circ} \mathrm{N}$ : implications concerning the mantle source composition for both seamount and adjacent EPR lavas. In Keating, B.H., Fryer, P., Batiza, R., and Boehlert, G.W. (Eds.), Seamounts, Islands, and Atolls. Geophys. Monogr., Am. Geophys. Union, 43:255-282.

Allan, J.F., Batiza, R., and Sack, R.O., 1994. Geochemical characteristics of Cocos Plate seamount lavas. Contrib. Mineral. Petrol., 116:47-61.

Allan, J.F., Chase, R.L., Cousens, B., Michael, P.J., Gorton, M.P., and Scott, S.D., 1993. The Tuzo Wilson volcanic field, NE Pacific: alkaline volcanism at a complex, diffuse, transform-trench-ridge triple junction. J. Geophys. Res., 98:22367-22387.

Allan, J.F., Sack, R.O., and Batiza, R., 1988. Cr-rich spinels as petrogenetic indicators: MORB-type lavas from the Lamont seamount chain, eastern Pacific. Am. Mineral., 73:741-753.

Arai, S., and Yurimoto, H., in press. Podiform chromitites of the Tari-Misaka ultramafic complex, southwestern Japan, as mantle-melt interaction products. Econ, Geol.

Basaltic Volcanism Study Project, 1981. Basaltic Volcanism on the Terrestrial Planets: New York (Pergamon Press).

Batiza, R., and Vanko, D., 1984. Petrology of young Pacific seamounts. J. Geophys. Res., 89:11235-11260.

Bender, J.F., Hodges, F.N., and Bence, A.E., 1978. Petrogenesis of basalts from the Project FAMOUS area: experimental study from 0 to $15 \mathrm{kbars}$. Earth Planet. Sci. Lett., 41:277-302.
Cannat, M., Bideau, D., and Hebert, R., 1990. Plastic deformation and magmatic impregnation in serpentinized ultramafic rocks from the Garrett transform fault (East Pacific Rise). Earth Planet. Sci. Lett., 101:216-232.

Carmichael, I.S.E., 1967. The iron-titanium oxides of salic volcanic rocks and their associated ferromagnesian silicates. Contrib. Mineral. Petrol., 14:36-64.

Dick, H.J.B., 1977. Evidence of partial melting in the Josephine Peridotite. In Dick, H.J.B. (Ed.), Magma Genesis: Proceedings of the American Geophysical Union Chapman Conference on Partial Melting in the Earth's Upper Mantle. Bull.-Oreg. Dep. Geol. Miner. Ind., 96:59-62. 1989. Abyssal peridotites, very slow spreading ridges and ocean ridge magmatism. In Saunders, A.D., and Norry, M.J. (Eds.), Magmatism in the Ocean Basins. Geol. Soc. Spec. Publ. London, 42:71-105.

Dick, H.J.B., and Bullen, T., 1984. Chromian spinel as a petrogenetic indicator in abyssal and alpine-type peridotites and spatially associated lavas. Contrib. Mineral. Petrol., 86:54-76.

Dick, H.J.B., and Fisher, R.L., 1984. Mineralogical studies of the residues of mantle melting: abyssal and alpine-type peridotites. In Kornprobst, J. (Ed.), Kimberlites II: The Mantle and Crust-Mantle Relationships: Amsterdam (Elsevier), 295-308.

Dick H.J.B., Fisher, R.L., and Bryan, W.B., 1984. Mineralogic variability of the uppermost mantle along mid-ocean ridges. Earth Planet. Sci. Lett., 69:88-106.

Dick, H.J.B., and Kelemen, P.B., 1991. Chromian spinel as a petrogenetic indicator of magmagenesis in shallow mantle rocks. Eos, 72:142.

Engi, M., 1983. Equilibria involving Al-Cr spinel; $\mathrm{Mg}-\mathrm{Fe}$ exchange with olivine; experiments, thermodynamic analysis, and consequences for geothermometry. Am. J. Sci., 283-A:29-71.

Engi, M., and Evans, B.W., 1980. A re-evaluation of the olivine-spinel Geothermometer: discussion. Contrib. Mineral. Petrol., 73:201-203.

Fisk, M.R., 1986. Basalt magma interaction with harzburgite and the formation of high-magnesian andesite. Geophys. Res. Lett., 13:467-470.

Francheteau, J., Armijo, R., Cheminée, J.L., Hekinian, R., Lonsdale, P.F., and Blum, N., 1990. 1 Ma East Pacific Rise oceanic crust and uppermost mantle exposed by rifting in Hess Deep (equatorial Pacific Ocean). Earth Planet. Sci. Lett., 101:281-295.

Gillis, K., Mével, C., Allan, J., et al., 1993. Proc. ODP, Init. Repts., 147: College Station, TX (Ocean Drilling Program).

Girardeau, J., and Francheteau, J., 1993. Plagioclase-wehrlites and peridotites on the East Pacific Rise (Hess Deep) and the Mid-Atlantic Ridge (DSDP Site 339) evidence for magma percolation in the oceanic upper mantle. Earth Planet. Sci. Lett., 115:137-149.

Hebert, R., Bideau, D., and Hekinian, R., 1983. Ultramafic and mafic rocks from the Garret transform fault near $13^{\circ} 30^{\prime} \mathrm{S}$. on the East Pacific Rise: igneous petrology. Earth Planet. Sci. Lett., 65:107-125.

Hekinian, R., Bideau, D., Francheteau, J., Cheminee, J.L., Armijo, R., Lonsdale, P., and Blum, N., 1993. Petrology of the East Pacific Rise crust and upper mantle exposed in the Hess Deep (eastern equatorial Pacific). $J$. Geophys. Res., 98:8069-8094.

Jacques, A.L., and Green, D.H., 1980. Anhydrous melting of peridotite at 0 $15 \mathrm{~kb}$ pressure and the genesis of tholeiitic basalts. Contrib. Mineral. Petrol., 73:287-310.

Jarosewich, E., Nelen, J.A., and Norberg, J.A., 1980. Reference samples for electron microprobe analysis. Geostand. Newsl., 4:43-47.

Kelemen, P.B., 1990. Reaction between ultramafic rock and fractionating basaltic magma. I. Phase relations, the origin of calc-alkaline magma series, and the formation of discordant dunite. J. Petrol., 31:51-98.

Kelemen, P.B., Whitehead, J.A., Aharonov, E., and Jordahl, K.A., in press. Experiments on flow focussing in soluble porous media, with applications to melt extraction from the mantle. J. Geophys. Res.

Kinzler, R.J., and Grove, T.L., 1992a. Primary magmas of mid-ocean ridge basalts, 1. Experiments and methods. J. Geophys. Res., 97:6885-6906.

1992b. Primary magmas of mid-ocean ridge basalts, 2. Applications. J. Geophys. Res., 97:6907-6926.

Klein, E.M., and Langmuir, C.H., 1987. Global correlations of ocean ridge basalt chemistry with axial depth and crustal thickness. J. Geophys. Res., 92:8089-8115.

Lago, B.L., Rabinowicz, M., and Nicolas, A., 1982. Podiform chromite ore bodies: a genetic model. J. Petrol., 23:103-125.

Leblanc, M., and Ceuleneer, G., 1992. Chromite crystallization in a multicellular magma flow: evidence from a chromitite dike in the Oman ophiolite. Lithos, 27:231-257.

Lonsdale, P., 1988. Structural pattern of the Galapagos microplate and evolution of the Galapagos triple junction. J. Geophys. Res., 93:13551-13574. 
Mazzullo, L.J., and Bence, A.E., 1976. Abyssal tholeiites from DSDP Leg 34: the Nazca Plate. J. Geophys. Res., 81:4327-4351.

McKenzie, D.P., and Bickle, M.J., 1988. The volume and composition of melt generated by extension of the lithosphere. J. Petrol., 29:625-679.

Nicolas, A., 1989. Structure of Ophiolites and Dynamics of the Oceanic Lithosphere: Dordrecht (Kluwer).

Niu, Y., and Batiza, R.. 1991. An empirical method for calculating melt compositions produced beneath mid-ocean ridges: application for axis and off-axis (seamounts) melting. J. Geophys. Res., 96:21753-21777.

Ozawa. K., 1983. Evaluation of olivine-spinel geothermometry as an indicator of thermal history for peridotites. Contrib. Mineral. Petrol., 82:5265 .

Perfit, M.R., and Fornari, D.J., 1983. Geochemical studies of abyssal lavas recovered by DSRV Alvin from eastern Galapagos Rift, Inca Transform, and Ecuador Rift, 2. Phase chemistry and crystallization history. J. Geophys. Res., 88:10530-10550.

Presnall, D.C., and Hoover, J.D., 1987. High pressure phase equilibrium constraints on the origin of mid-ocean ridge tholeiites. J. Petrol., 20:3-35.

Roeder, P.L., Campbell, I.H., and Jamieson, H.E., 1979. A re-evaluation of the olivine-spinel geothermometer. Contrib. Mineral. Petrol., 68:325334.

Sack, R.O., 1980. Adirondack mafic granulites and a model lower crust: summary, Geol. Soc. Am. Bull., 91 (Pt. 1):89-93.
1982. Spinels as petrogenetic indicators: activity-composition relations at low pressures. Contrib. Mineral. Petrol., 79:169-186.

Sack, R.O., Carmichael, I.S.E., Rivers, M.L., and Ghiorso, M.S., 1980. Ferric-ferrous equilibria in natural silicate liquids at $1 \mathrm{bar}$. Contrib. Mineral. Petrol., 75:369-376.

Sack, R.O., and Ghiorso, M.S., 1991a. An internally consistent model for the thermodynamic properties of $\mathrm{Fe}-\mathrm{Mg}$-titanomagnetite-aluminate spinels. Contrib. Mineral. Petrol., 106:474-505.

, 1991b. Chromian spinels as petrogenetic indicators: thermodynamics and petrological applications. Am. Mineral., 76:827-847.

Scowen, P.A., Roeder, P.L., and Helz, R., 1991. Reequilibration of chromite within Kileauea Iki lava lake, Hawaii. Contrib. Mineral. Petrol., 107:820.

Sinton, J., 1977. Equilibration history of the basal alpine-type peridotite, Red Mountain, New Zealand. J. Petrol., 18:216-246.

Walter, M.J., and Presnall, D.C., 1994. Melting behavior of simplified therzolite in the system $\mathrm{CaO}-\mathrm{MgO}-\mathrm{Al}_{2} \mathrm{O}_{3}-\mathrm{Na}_{2} \mathrm{O}$ from 7 to 35 kbar. J. Petrol., $35: 329-359$.

Date of initial receipt: 1 August 1994

Date of acceptance: 21 February 1995

Ms 147SR-009 

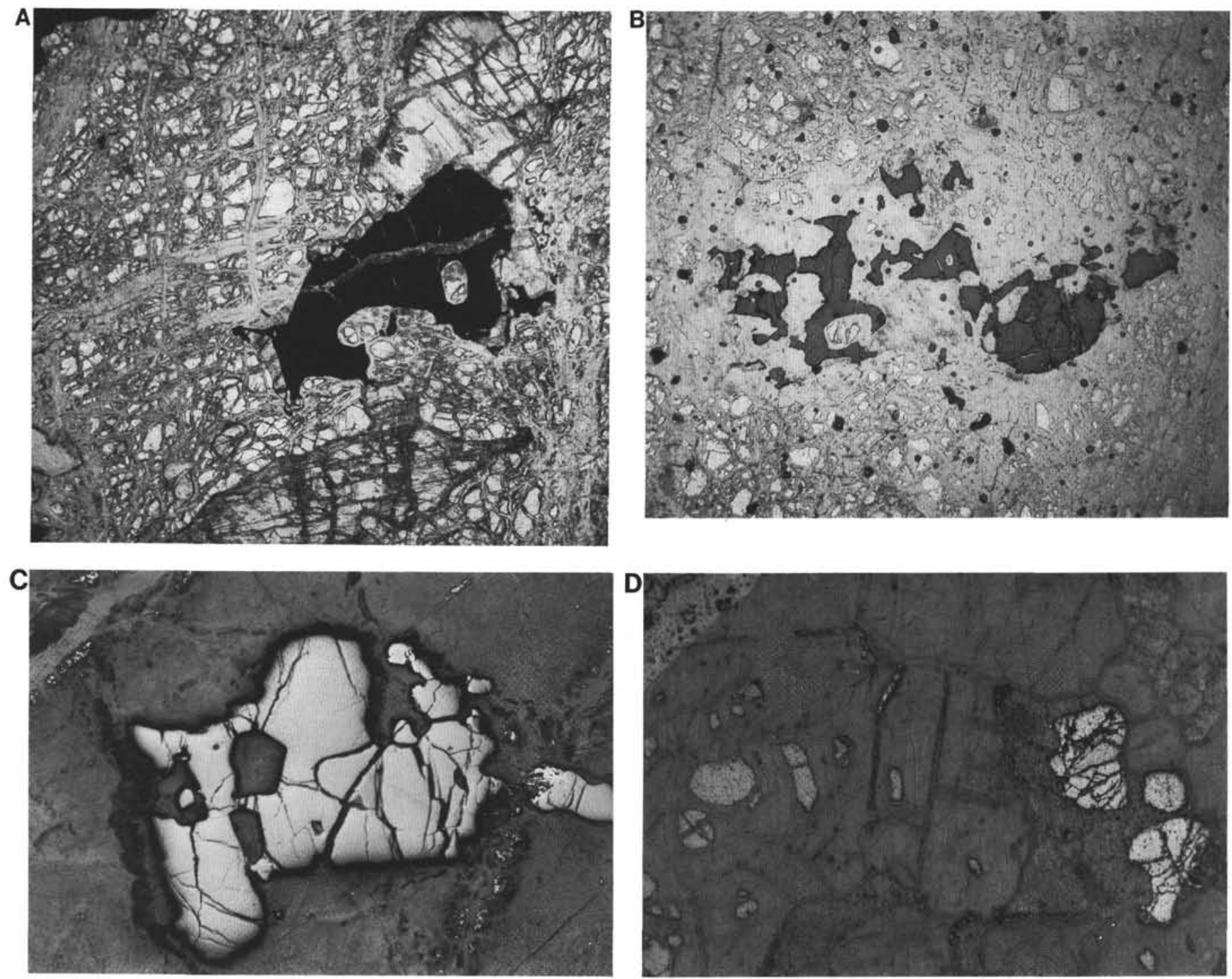

Plate 1. A. Photomicrograph ( $2.1 \mathrm{~mm}$ across, in transmitted light) of blocky, anhedral Cr-spinel in harzburgite Sample 147-895D-5R-1, 83-86 cm. Note also the presence of orthopyroxene porphyroclasts and smaller, anhedral grains of clinopyroxene attached to the spinel margin. Analyses of this spinel are given by transect $\mathrm{Cl}$ in Figure 6 and analysis $\mathrm{Cl}$-C in Table 2. B. Photomicrograph (2.8 mm across, in transmitted light) of holly-leaf $\mathrm{Cr}$-spinels in harzburgite Sample 147-895C-4R-1, 10-12 cm. Note the much greater alteration of this sample than that shown in Figure I. Analyses of these spinels are given by transects Al and A2 in Figure 6 and analyses A1-C, A1-R, and A2-C in Table 2. C. Reflected light photomicrograph (3.5 mm across) of irregular, rounded Cr-spinel in dunite Sample 147-895D-9R-1, 101-105 cm. Analyses of this spinel are given by transects Al and A2 in Figure 6 and analyses A1-C, A2-C, and A2-R in Table 2. D. Combination reflected- and transmitted-light photomicrograph (3.5 mm across) of irregular, rounded $\mathrm{Cr}$-spinel in dunite Sample 147-895E-6R-3, 108-111 cm. Analyses of this spinel are given by transect B1 in Figure 6 and analysis B1-C in Table 2. 

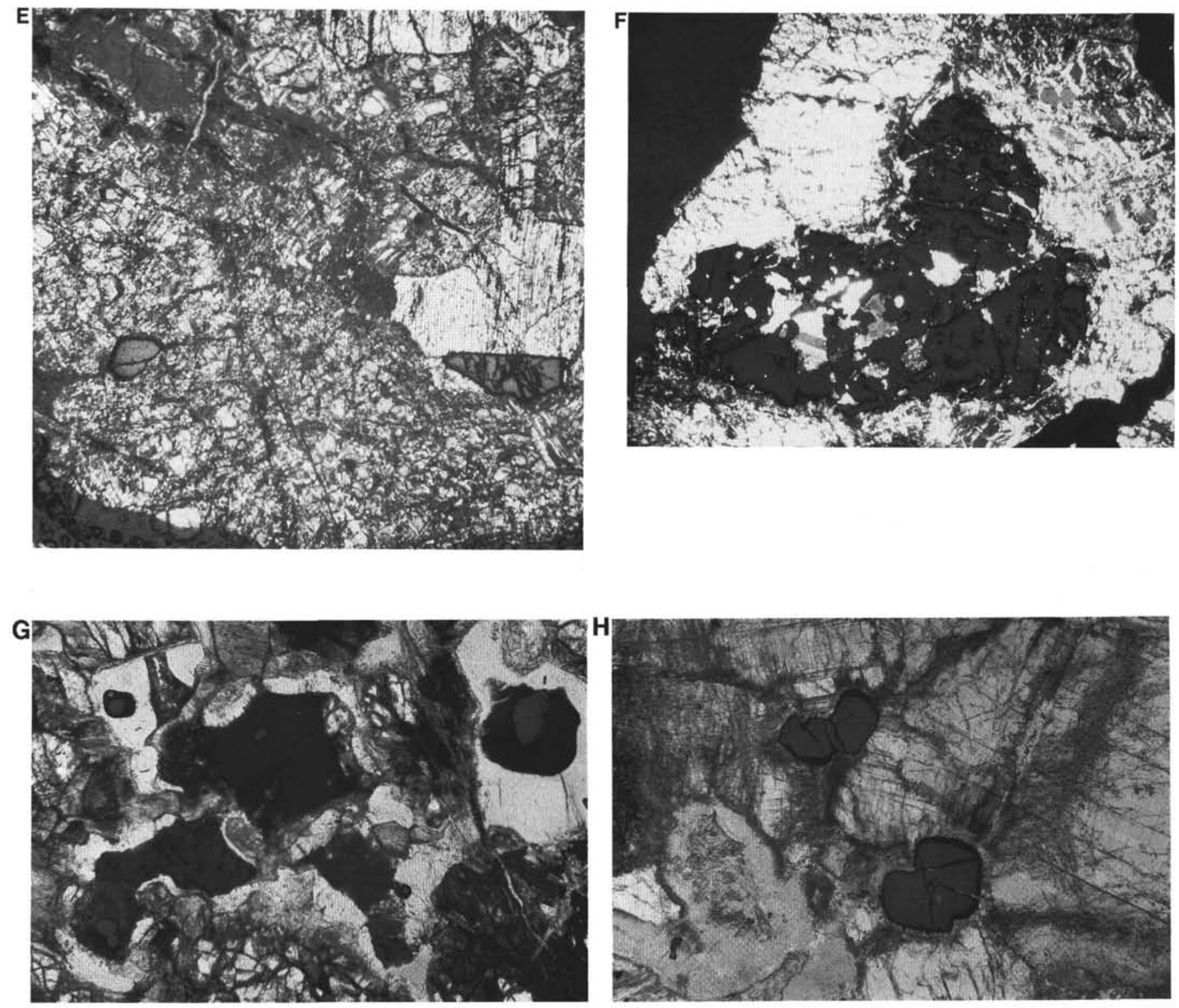

Plate 1 (continued). E. Combination reflected-and transmitted-light photomicrograph (1.9 mm across) of subhedral, rounded Cr-spinel in troctolite Sample 147 $895 \mathrm{C}-4 \mathrm{R}-1,63-68 \mathrm{~cm}$. Melt segragations are represented by irregular patches of clinopyroxene surrounded by olivine. Spinel occurs as a grain on the edge of a melt segregation and as an isolated grain surrounded by olivine (represented by analyses 7-C and 8-C, respectively, in Table 2). F. Combination reflected- and transmitted-light photomicrograph ( $4.5 \mathrm{~mm}$ across) of skeletal $\mathrm{Cr}$-spinel surrounded by olivine from troctolite Sample 147-895C-4R-2, 75-78 cm, and represented by analyses in transect $\mathrm{Cl}$ in Figure 6 and analyses $\mathrm{C} 1-\mathrm{C}$ and $\mathrm{C} 2-\mathrm{C}$ in Table 2. Note the plagioclase and clinopyroxene inclusions within the spinel; these are represented by analyses in Table 4 . G. Combination reflected- and transmitted-light photomicrograph ( $1.75 \mathrm{~mm}$ across) of rounded, subhedral Cr-spinel inclusions in plagioclase that has been altered to chlorite, clays, and hydrogrossular (dark patches) and to serpentine and tremolite (surrounding lighter area) (Gillis, Mével, Allan, et al., 1993). These occur within a melt segregation of olivine gabbro Sample 147-895D-7R-2, 71-75 cm. The large spinel inclusion at the top of the photograph is represented by transect D1 of Figure 6 and by analysis D1-IPC in Table 2; the spinel inclusion at the lower right is represented by analysis 28-IPC in Table 2. Note the clusters of $\mathrm{Fo}_{83-84}$ olivine grains (Table 3; light, heavily fractured grains). $\mathrm{H}$. Combination reflected- and transmitted-light photomicrograph (1.75 mm across) of subhedral $\mathrm{Cr}$-spinel inclusions in partially altered plagioclase within olivine gabbro Sample 147-895E-2R-1, 36-39 cm. The larger grain is represented by transect B1 in Figure 6 and by analyses B1-IPC and B1-IPR in Table 2. 